\title{
The germs inside me: Pediatric patients' conceptualization of illness and regulation of emotions during hospitalization
}

\author{
Sarah E. Buck
}

West Virginia University

Follow this and additional works at: https://researchrepository.wvu.edu/etd

\section{Recommended Citation}

Buck, Sarah E., "The germs inside me: Pediatric patients' conceptualization of illness and regulation of emotions during hospitalization" (2014). Graduate Theses, Dissertations, and Problem Reports. 136. https://researchrepository.wvu.edu/etd/136

This Thesis is protected by copyright and/or related rights. It has been brought to you by the The Research Repository @ WVU with permission from the rights-holder(s). You are free to use this Thesis in any way that is permitted by the copyright and related rights legislation that applies to your use. For other uses you must obtain permission from the rights-holder(s) directly, unless additional rights are indicated by a Creative Commons license in the record and/ or on the work itself. This Thesis has been accepted for inclusion in WVU Graduate Theses, Dissertations, and Problem Reports collection by an authorized administrator of The Research Repository @ WVU. For more information, please contact researchrepository@mail.wvu.edu. 
The Germs inside Me: Pediatric Patients' Conceptualization of Illness and Regulation of Emotions during Hospitalization

\author{
Sarah E. Buck \\ Thesis submitted to the \\ College of Education and Human Services \\ at West Virginia University \\ in partial fulfillment of the requirements for the degree of
}

\begin{abstract}
Master of Arts
In

Educational Psychology

with an emphasis in Child Development and Family Studies
\end{abstract}

Suzanne C. Hartman, Ph.D., Chair

Christina Duncan, Ph.D.,

Amy Kennedy Root, Ph.D., Jessica Troilo, Ph.D.,

Department of Learning Sciences and Human Development

Morgantown, West Virginia

2014

(C) 2014 Sarah E. Buck 


\title{
Abstract \\ THE GERM INSIDE ME: PEDIATRIC PATIENTS' CONCEPTUALIZATION OF ILLNESS AND REGULATION OF EMOTIONS DURING HOSPITALIZATION
}

\author{
by Sarah E. Buck
}

The transition into a medical setting can be difficult for children under the age of 18 . Previous research has identified a child's capacity to construct accurate representations of illness, in addition to the construction and application of adaptive strategies to modify emotional arousal, as significant predictors of successful adjustment to a medical environment. Preexisting research has neglected to investigate the constructs of illness conceptualization and regulatory systems of emotions. Using a sample of 26 pediatric patients and 26 adult informants, the current study explored the complex relationship between emotional regulation and the conceptualization of illness among children, from the ages of three to five, in the management of a hospital setting. Measures of inhibition, attentional shift and inhibitory self-control were significantly and negatively related to general illness conceptualization. In addition, the study investigated the implications of increased exposure to hospitalization on pediatric patients' development of health-related knowledge and strategies to regulate emotional arousal in early childhood. Contrary to prediction, children characterized as experiencing frequent admittances to a medical setting attained significantly higher means scores, reflecting poor aptitude, on measures of attentional shift, emotional control, and flexibility. The present investigation validates the necessity of respective therapeutic models to appropriately intervene for vulnerable, medically fragile populations 


\section{Table of Contents}

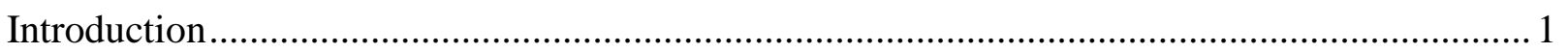

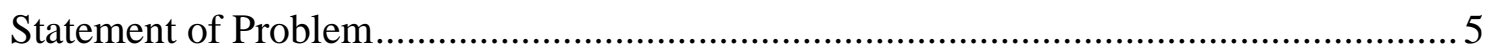

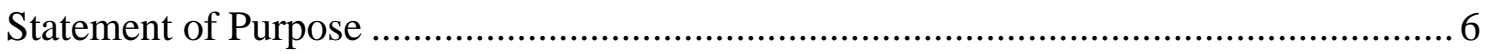

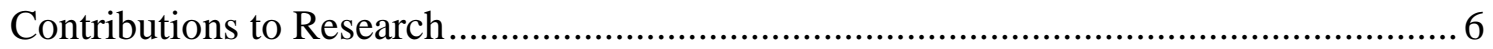

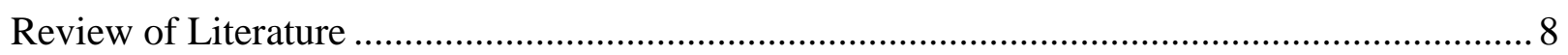

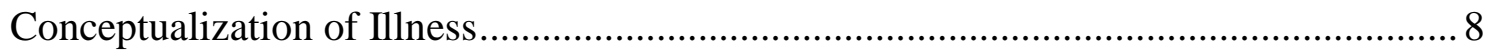

Piaget's Cognitive Developmental Stages ................................................................ 9

Piaget's Model of Cognitive Development and Illness Causality .................................... 11

Enhanced Understanding of Illness Causality ……………....................................... 16

Emotional Appraisal of Hospitalization....................................................................... 18

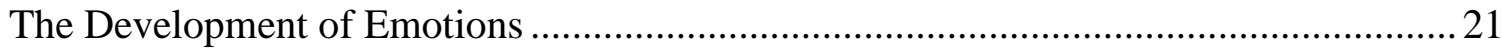

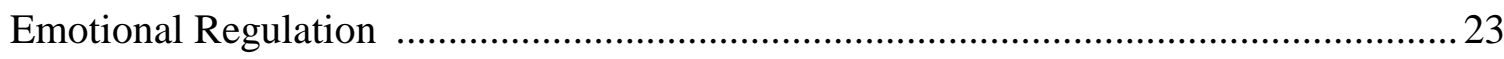

The Developmental Trends of Emotional Regulation .......................................................24

Cognitive Implications of Emotional Development ………......................................28

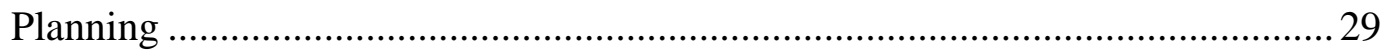

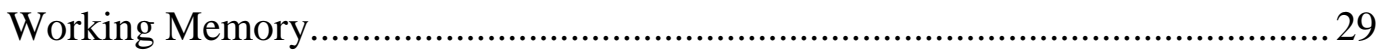

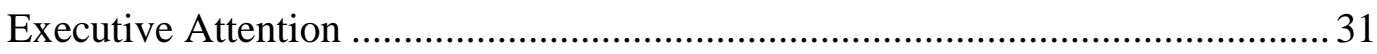

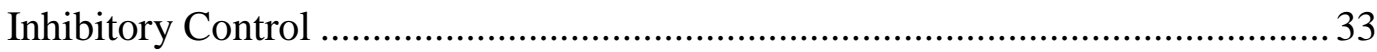

Integration of Emotional and Cognitive Processing ........................................................ 34

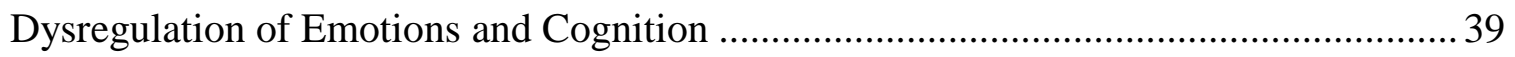

Impact of Prior Hospitalization................................................................................ 42

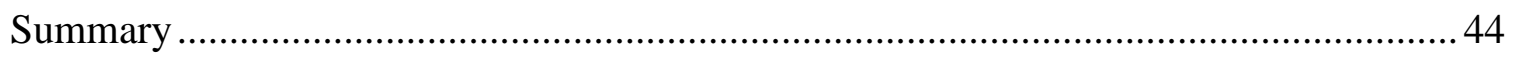




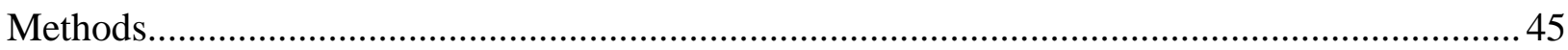

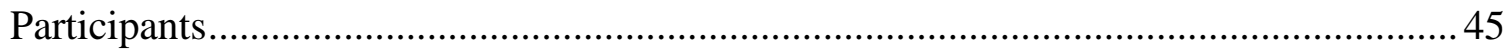

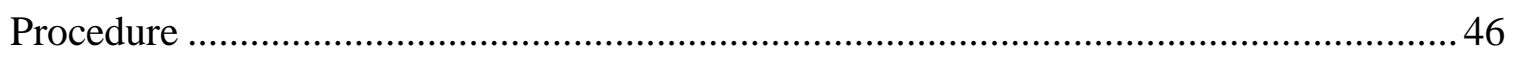

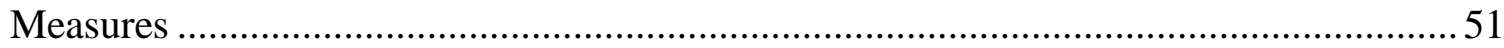

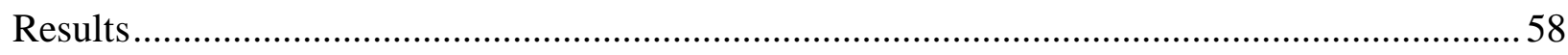

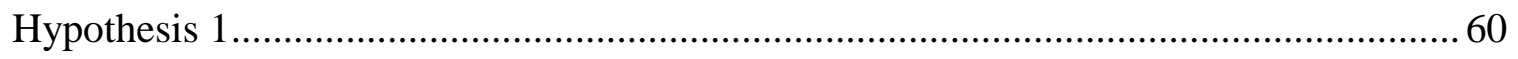

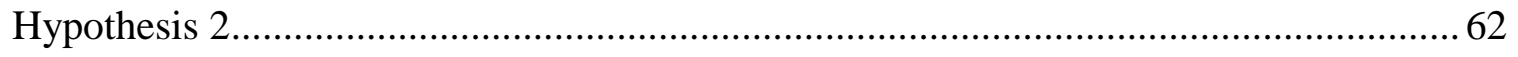

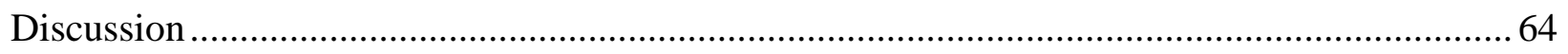

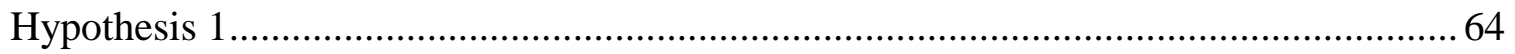

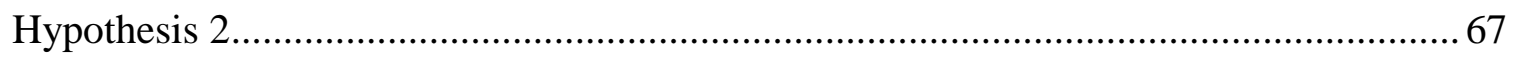

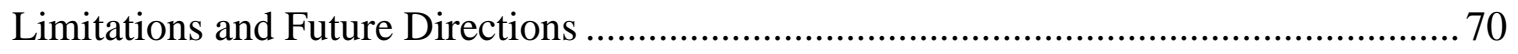

Child Characteristics ................................................................... 70

Methodological Considerations ...................................................... 75

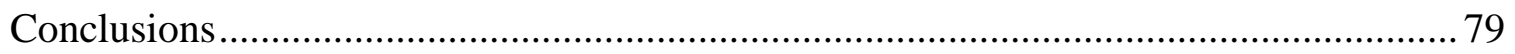

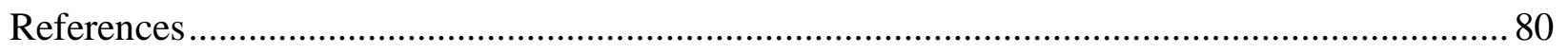




\section{List of Tables}

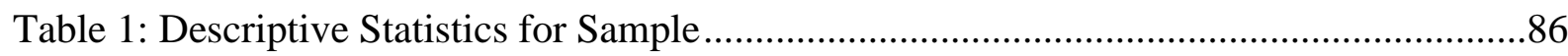

Table 2: Descriptive Statistics for General Illness and Behavioral Rating by Gender ...........88

Table 3: Descriptive Statistics for Behavioral Rating Inventory T-values ...........................88

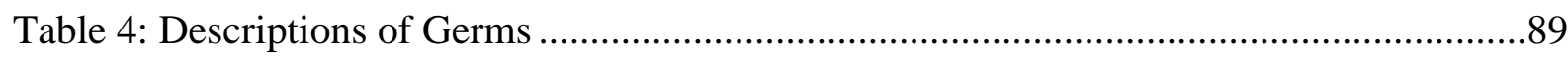

Table 5: Pearson Correlation Variables for Interest ..................................................... 90

Table 6: Independent Sample T Test for Initial and Frequent Exposure ..............................91

Table 7: General Illness Concept Interview Classifications ............................................992 


\section{List of Figures}

Figure 1: Total Hospital Admittances for Pediatric Participating Patients............................93

Figure 2: Extent of Exposure to Hospitalization Histogram ............................................94

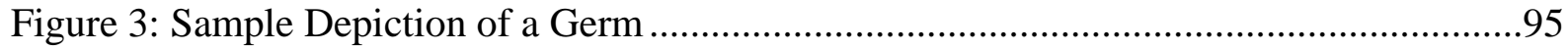

Figure 4: Supplemental Sample Depiction of a Germ .................................................. 96 


\section{List of Appendices}

Appendix A: General Demographic Questionnaire .......................................................99

Appendix B: The Behavioral Rating Inventory of Executive Function-Preschool ................98

Appendix C: The General Illness Concept Interview .................................................100

Appendix D: Subset Banks Questionnaire ................................................................102 


\section{Chapter I}

\section{Introduction}

Hospitalization can be a dramatic process. For the purpose of this study, hospitalization can be defined as a duration in time in which an individual receiving medical care is confined to a hospital for least an overnight stay. For patients under the age of 18, or pediatric patients, an unfamiliar location, limitations in mobility, debilitating symptoms, medical procedures and lack of independence can all be distressing (Rokach, 2010). Very often, pediatric patients have difficulty appropriately adjusting to this transition. Pediatric patients' knowledge of illness and their ability to integrate this information is critical to appropriately transition through various medical settings. The conceptualization of illness can be organized into a process of defining features, identifying the causes, acknowledging consequences, and recognizing a recovery period (Paterson, Moss-Morris, \& Butler, 1999). However, children's representations of illness vary at different periods in development.

The theoretical framework presented by Piaget (1969) provides a foundation for the critical analysis of children's perceptional understanding of illness. In Piaget's model of cognitive development, children's cognitive capacities are organized into developmental stages. Each period of development is marked by advancements in cognitive reasoning (Piaget \& Inhelder, 1969). As a child advances through each stage, naïve interpretations develop into more sophisticated cognitive appraisals. This structure of cognitive maturation resembles the developmental variations in the conceptualization of illness (Borzekowski, 2009).

As children progress through the cognitive developmental periods, terms used to identify and describe illness evolve from external features to internal processes. Prior to the age of two, children acknowledge the sensations associated with feeling ill (Koopman, Baars, Chaplin, \& 
Zwindermann, 2004). This period, described as the sensorimotor stage, reflects an infant's immediate physical states.

As a child progresses to the preoperational stage, from the ages of two to six, illness is often described by physical appearances or observable actions (Borzekowski, 2009; Koopman et al., 2004). As children mature, they move from believing that illness is caused by external forces to concepts of standard medical and cultural explanations. Concepts of contagion and contamination begin to be introduced into their explanations of illness. Young children rely heavily on naïve biological beliefs of illness. This naïve germ theory of contagion focuses on the transmission of illness through germs (Williams \& Binnie, 2002). Very often, incorrect mechanisms are frequently assigned to non-contagious ailments. In the developmental stages, theories of illness causality are misapplied to non-contagious illnesses as a result of poor biological understanding.

In middle childhood, or in the concrete operational stage, children are capable of describing illness as it relates to particular behaviors, consequences, and associated symptoms. In the final cognitive period, the formal operational stage, from the ages of 7 to 11 , physiological explanations of illness correspond to explain beliefs of infection, maintenance of health, and treatment options (Myant \& Williams, 2005). Research presented in the review of literature will further demonstrate the well-defined developmental progression of children's comprehension of illness, with emphasis placed on the periods of preoperational development, as it corresponds to Piaget's cognitive developmental theory.

Children's comprehension of their illness is an integral aspect of their medical experience. By developing an expansive understanding of the source, sequence, and period of recovery, pediatric patients can effectively manage the medical setting. Enhanced understanding 
of illness can reduce a child's level of stress and encourage self-management and compliance with medical procedures (Chilman-Blair, 2010; Eiser \& Havermans, 1992; Levetown, 2008; Perrin \& Gerrity, 1981; Whaley, 2000). By acquiring extensive understanding of health-related knowledge, pediatric patients can access a stable framework to manage hospitalization.

The emotions experienced by pediatric patients often reflect ambiguity and apprehension in their evaluation of the medical environment (Rokach, 2010). Doctor's exams, unfamiliarity, lack of information, separation from loved ones, and restriction in activities can evoke an array of emotional reactions. The manner in which an individual adjusts to emotional states is known as emotional regulation (Denham, 1998). This process is responsible for monitoring, appraising, and modifying the experience of emotions through behavioral, cognitive, and emotional strategies (Denham, 1998).

As a child acknowledges the feelings experienced, he or she is able to manage the situation in perceptual ways. The construction of flexible methods to redirect emotional arousal is a subjective evaluation of relevant features and available resources to manage of a significant experience (Claar, Walker, \& Smith, 2002; Jaanister, Hayes, \& von Baeyer, 2007). This process of appraising one's perceived ability to manage emotional events is central to the regulation of affective states. Behavioral, cognitive, and emotional efforts to manage external and internal demands that are appraised as demanding or beyond the available resources of the individual develop as regulatory processes of emotions (Boekaerts \& Roder, 1999; Denham, 1998). Children initially rely on behavioral approaches in managing experienced emotions. For preschool-aged children, from the ages of three to five, the entrance into a primary educational setting and advances in language and independence fosters enhanced regulatory strategies of 
emotions. With age, children substitute behavioral responses for self-guided, cognitive and emotional regulatory strategies.

The capacity to develop and implement effective strategies of emotion regulation can contribute to the management of emotionally eliciting encounters. An emotionally eliciting experience, or event, can be described as a subjective appraisal of an encounter that evokes the expression of emotional states (Thompson, 1991). Emotionally eliciting events can provoke an array of emotional states, such as happiness, sadness, fear, and excitement.

By altering affective states, children can direct behavioral outcomes (Denham, 1998). However, applications of effective regulatory strategies develop in progression with cognitive resources. Characteristics of cognition that facilitate the organization of information, preservation of attention, and experience of planful outcomes are synonymous with the regulation of emotions (Vohs \& Baumeister, 2011). Cognitive processes of inhibitory control, attention shifting, working memory, and planning, operate as a cohesive system to facilitate the management of emotional arousal.

For pediatric patients, a medical setting can be an emotional eliciting environment. However, with increased experience with illness and frequent exposure to medical encounters, pediatric patients develop adaptive logic in understanding illness causality and in addressing emotions experienced during hospitalization (Case et al., 2005; Crisps et al., 1996; Kopp, 1989; Paterson et al., 1999; Williams \& Binnie, 2002). As children cognitively mature and practice mechanisms for managing emotion-eliciting situations, they become more competent in adjusting arousal. Once effective mechanisms for regulating emotions are established, such strategies are employed regularly with increased exposure (Kopp, 1989). 
Children also develop more sophisticated representations of illness with experience (Williams \& Binnie, 2002). Pediatric patients with elevated levels of exposure to medical experiences, such as the chronically ill, retain more detailed conceptual representations of illness (Case, Fertig, \& Paxson, 2005; Crisps, Ungerer, \& Goodnow, 1996; Paterson et al., 1999). With increased experience, pediatric patients develop and apply cognitive representations of the source, progression, and treatment of illness.

For pediatric patients, comprehensive knowledge regarding health, the development of adaptive strategies to redirect emotionally eliciting experiences, and accessible resources to manage a medical experience are formative components in successful management of a medical setting. The current study examined the relationship between emotional regulation and the conceptualization of illness in preschool-aged pediatric patients, from three to five years. As a transitional period in intellectual, expressive and social development, the age of interest served as a portal into the progression of regulatory processes of emotion and cognitive maturation.

\section{Statement of Problem}

The conditional demands of a medical setting can determine an individual capacity to effectively exercise functions of cognition and emotional regulatory processes. For pediatric patients, persistent emotional arousal in a medical environment can impair cognitive and emotional resources necessary to develop accurate representation of illness and apply adaptive regulatory strategies of emotions (Fox, 1994; Spirito, Stark, \& Typ, 1994).

Prior studies have demonstrated young children's inadequate knowledge regarding the origins, progression, and recovery periods of illness as a result of restrictions in the processing of information. Competing cognitive resources can become overwhelmed because of the emotional complexity of a medical environment. The obstruction of a pediatric patient's capacity to manage 
information concerning health-related material results in confounded representations of illness, thus undermining an individual's perceived management of emotional states (Myant \& Williams, 2005). These pediatric patients are often vulnerable to the development of dysregulated emotional processes (Fox, 1994).

The ability to develop knowledgeable representations and apply flexible methods to adjust emotional arousal can modify a pediatric patient's appraisal and management of a medical experience. For pediatric patients with increased experience with illness and reoccurring exposure to medical encounters, constructive cognitive and emotional resources can be observed. Prior research has identified the development of adaptive logic in establishing representations of illness and in directing the experience of emotions in pediatric patients with previous exposure to medical settings (Case et al., 2005; Crisps et al., 1996; Kopp, 1989; Paterson et al., 1999; Williams \& Binnie, 2002). This reasoning justified the study's need to investigate the relationship between emotional regulation and the conceptualization of illness of pediatric patients from the ages of three to five with increased exposure to hospitalization. As supported in the presented literature, the age of interest is a critical period in language acquisition, cognitive maturation and social competence. Therefore, the restriction in participants' age assisted in exploring the developmental progression of regulatory processes of emotion and illness conceptualization.

\section{Statement of Purpose}

To effectively manage an emotionally eliciting, contextually complex experience of hospitalization, pediatric patients must access flexible resources to develop appropriate healthrelated knowledge in addition to adaptive regulatory processes. The purpose of the current study was to address the dynamic nature between emotional regulation and the conceptualization of 
illness of hospitalized pediatric patients from the ages of three to five. Specifically, the purpose of this research study was to: (1) assess pediatric patients' regulation of emotions; (2) identify pediatric patients' understanding of illness; and (3) evaluate the implications of exposure to a hospital setting on the dependent variables.

\section{Contributions of this Research}

In recent years, the field of medicine and child psychology has attempted to recognize the emotional experience and implication of hospitalization on pediatric patients. Much of the relevant literature has focused on advocating for children's psychological well-being by identifying emotionally eliciting features of medical settings, children's cognitive representations of illness, and successful approaches to managing emotional arousal. However, such investigations have failed to integrate constructs of illness conceptualization, and regulatory systems of emotion as a collaborative relationship. This study offered insight into how pediatric patients from the ages of three to five comprehend illness, as well as the interplay of this compression with the adaption to emotions experienced during hospitalization. By delineating the relationship between children's cognitive illness representations and emotional adjustment to illness, medical professionals may be encouraged to promote adaptive emotion regulatory strategies and correct conceptions of illness. When incorporating such practices into the medical field, the successful management of medical environments for pediatric patients may be facilitated. 


\section{Chapter II}

\section{Review of the Literature}

To successfully manage the experience of hospitalization, pediatric patients must develop appropriate health-related knowledge as well as access flexible emotional regulatory processes to manage arousal. The objective of this chapter is to review relevant literature pertaining to this research study. Following the theoretical contributions of Piaget's cognitive developmental model, the chapter considers relevant literature reflecting the progression of health-related knowledge. The detailed analysis of literature will emphasize the preoperational stage in cognitive development, as it is the age of interest for the research investigation. A comprehensive evaluation of the implications of accurate representations of health on pediatric patients' emotional appraisals of medically relevant encounters will be explored. To adequately adjust to an expressive environment, the integration of cognitive control processes to facilitate the management of emotional arousal will be discussed. To conclude, the assessment of literature will evaluate the implications of increased exposure to the emotionally confounding medical setting on the ability to develop compressive knowledge and exercise accommodating emotion regulatory strategies.

\section{The Conceptualization of Illness}

The development of health-related knowledge and the capacity to assimilate information can direct a child's medical experience. The conceptualization of illness can be organized into a process of defining the characteristics of illness, identifying consequences, recognizing the temporal course of an illness, distinguishing between the causes, and acknowledging a recovery period (Paterson et al., 1999). Children's explanations of illness, however, vary considerably at different periods of development. 


\section{Piaget's Cognitive Developmental Stages}

The theoretical model presented by Piaget (1969) provides a foundation for the critical analysis of children's perceptional understanding of illness. In Piaget's model of cognitive development, children's knowledge follows a progression of developmental stages. Each period of development is identified by expansions in cognitive reasoning. From the time of birth, through approximately the second year of life, children experience their external environment through proximal sensations (Piaget \& Inhelder, 1969). This period in cognitive development is known as the sensorimotor stage. Through repetitive exposure to effortless reflexive behaviors, routines are developed (Cowen, 1979; Piaget \& Inhelder, 1969).

Instinctual sensory responses begin to develop into sophisticated, purposeful actions following the second year. From the ages of two to six, children begin to develop associations between their precise actions and the resulting outcomes as they progress into the preoperational phase of cognition. By directly collaborating with the environment, children can harness opportunities to develop conceptual symbols (Piaget \& Inhelder, 1969). The creation of pragmatic mental representations supports and guides the experiences of the child (Cowen, 1979; Piaget \& Inhelder, 1969). This mental progression is influenced by the expansion of the child's immediate environment in early childhood. The development of advanced motor skills, increased opportunities to engage in social experiences, and expanding linguistic abilities, encourages the development of internal representations. For example, with increased exposure and developing linguistic skills, children are encouraged to develop mental representations of health and illness by reflecting on experiences. This period of perceptual construction reflects external, egotistic experiences of the individual (Piaget \& Inhelder, 1969). 
The perspectives of others are incorporated into an individual's perceptual understanding with the progression of concrete operational thought. This stage in cognitive growth extends from age seven to approximately eleven. After the age of seven, children's reduction in egotistic reasoning fosters an individual's effective understanding of the internal mechanisms of others (Cowen, 1979; Piaget \& Inhelder, 1969). The ability to acknowledge outside perspectives can increase a child's knowledge and understanding of health and illness. Health-related conditions exclusive to the child's immediate environment can be explored from altered perspectives. However, abstraction of complex conceptual information cannot be successfully recognized at this period in cognitive development.

As a child advances to formal operational thinking, abstract concepts, such as peace and justice, can be understood and organized into an individual's perceptual knowledge (Piaget \& Inhelder, 1969). This phase in cognitive maturation develops in early adolescence, from the ages of 12 to approximately 18 . Additional abstract thought is recognized as an adolescent is able to hypothesize the arrangement of experiences through implicit cues in the environment (Cowen, 1979; Piaget \& Inhelder, 1969). An adolescent may produce multiple explanations for a solitary event to derive appropriate conclusions. For example, an adolescent may develop varying opinions in regards to the source of an illness. This development of advanced cognitive evaluations permits an adolescent to examine events beyond physical characteristics (Cowen, 1979; Piaget \& Inhelder, 1969). Sophisticated rationalizations, such as an illness occurring as a genetic mutation, may be selected rather than physical forces. Sources beyond concrete observable events can be inferred. Implicit conclusions can then be deduced. 
With the progression of each stage, naïve interpretations develop into more sophisticated cognitive appraisals. This organization of cognitive growth resembles the developmental variations in the conceptualization of illness (Borzekowski, 2009).

\section{Piaget's Model of Cognitive Development and Illness Causality}

From infancy to adolescence, children's perceptional understanding of illness can be arranged into a systematic progression of cognitive development (Bibace \& Walsh, 1980). Attaining appropriate knowledge concerning the source, progression, and treatment of illness varies considerably at distinct periods of development.

In the sensorimotor stage, children under the age of two identify and explore their surroundings through physical sensory engagement with the environment (Piaget \& Inhelder, 1969). During this period, children prior to the age of two acknowledge sensations as the primary association with feeling ill (Koopman et al., 2004). Specifically, properties of the individual's physical well-being, such as feeling feverish or warm, are initial sensations expressed. Physical limitations or restrictions in activities are also used from to define illness (Redpath \& Rogers, 1984). A pediatric patient in the sensorimotor stage may describe illness as restricting their ability to play freely. In addition, children operating at the sensorimotor stage describe ambiguous emotions, such as, sadness in relation to the experience of feeling poorly (Borzekowski, 2009). For a pediatric patient under the age of two, thoughts of illness may evoke sadness, resulting in sulking and tears. However, as a result of limitations in linguistic abilities, the true experience of feeling ill is unexplored (Borzekowski, 2009).

As a child advances to the preoperational stage, illness is defined by physical and observable actions (Borzekowski, 2009; Koopman et al., 2004). This period in cognitive maturation is characterized by the development of causal inferences. As a result, preoperational 
children regularly regard themselves as the cause of an illness (Kister \& Patterson, 1980;

McIntosh, Stephens, \& Lyons, 2013; Redpath \& Rodgers, 1984). This erroneous appraisal is known as immanent justice. Immanent justice, first developed by Piaget (1969), is the belief that two events occurring simultaneously are causally related. For example, a child may hurt their sibling and the following day contract a cold. Although the experiences are unrelated, the child may determine poor behavior to prompt the development of an illness. Based on the concept of immanent justice, pediatric patients functioning at a preoperational level often identify disobedience as the source of illness (Kister \& Patterson, 1980).

Children from the ages of two to six also determine the transmission of an ailment as initiated by an external physical phenomenon that coincides with the physical symptoms (Whaley, 2000). Indication of weather conditions are frequently referenced (Myant \& Williams, 2005). Symptoms reflecting a cold climate, such as a runny nose or chills, correspond with descriptions of the causality of illness. However, at this time, preoperational children are incapable of describing the implications of physical forces on internal processes (Whaley, 2000). A child operating at this developmental period cannot comprehend the sequence from the cause to the physical effects. Children under the age of six cannot reason in what ways an external phenomenon can develop into an illness. The understanding and knowledge of physiological and biological mechanisms of transmitting an ailment are unsophisticated at this time in cognitive development.

Prior to the age of seven, children rely heavily on naïve biological beliefs of illness. The naïve germ theory focuses on the physical nature of germs in the transmission of illness (Williams \& Binnie, 2002). Biological concepts of contagion and contamination begin to be introduced into children's explanations of illness at the preoperational stage of development. The 
physiological process of contamination involves the transmission of impurities through contact with an object (Williams \& Binnie, 2002). During the preoperational stage, children are capable of providing relevant contamination explanations, identifying germs and invisible contaminants in their description of illness causation (McIntosh et al., 2013). For example, a child may describe acquiring a cold after playing with an unclean toy. Contagion, in contrast, describes a complex sequence by which contaminants are transmitted between people. Concepts of contagion, revealed in the preoperational stage of development, can be demonstrated in pediatric patient's rationalization of developing an illness after coming in contact with an ill peer. Although as early as age four children are capable of referring to principles of contamination and contagion, comprehension of primary biological processes are underdeveloped (Myant \& Williams, 2005). Children under the age of seven describe the period of time from exposure to a virus, to the appearance of symptoms as an immediate causal event (Myant \& Willams, 2005). This elaboration in the period of time demonstrates the lack of knowledge in the reproduction of viruses.

Very often, as a consequence of misconstrued biological mechanisms, children under the age of seven integrate incorrect mechanisms to non-contagious ailments (Myant \& Williams, 2005). Younger children frequently overextend the concept of contingency to inappropriate ailments. General knowledge of common ailments and inaccurate theories of biological processes are often misused or applied excessively to varying situations. Conditions that do not result from contamination, as well as injuries from an accident, are often incorporated into this thought process (Kister \& Patterson, 1980). For example, a child functioning at the preoperational period in development may determine the source of appendicitis to be the result of 
contaminated food. The accuracy of knowledge concerning biological principles of contagion and contamination does not develop until later ages.

Into middle childhood, from the ages of 7 to 12 , children's representation of illness evolves considerably. Detailed explanations of illness develop as children are able to distinguish between internal and external characteristics at the concrete level (Bibace \& Walsh, 1980; Whaley, 2000). This indication of cognitive maturity from the age of seven to twelve is a reflection of concrete operational reasoning. With age, the cause of the illness and the methods of transmission become discernible. The source of illness is regarded as an internalized process within the body as a consequence of external conditions. The causal force of the illness must gain access through internalization of the agent. Pediatric patients within the concrete operational stage frequently render methods of ingestion and inhalation as the means of internal access (Whaley, 2000). The sequence of instrumental forces reveals children's expanding understanding of biological principles.

Although limited in accurate knowledge of anatomical processes, children's progression in concrete-operational thought is further demonstrated by the development of health conscious behaviors. Attention to health practices are initially communicated through sociocultural interactions (McIntosh et al., 2013). Opportunities to develop wellness guidelines are established through educational and social instructions. Elementary methods of prevention, such as frequent washing of hands and shielding a cough, strengthen behavior al rules related to contamination and contagion. Limitations in abstract thought and socially constructed wellness practices reinforce already narrow understanding of the biological construction of illness. Not until abstraction in thought is well-established can children transcend predetermined external inferences. 
The preconceptions maintained by children regarding the course of illness are reconstructed once more in adolescence. This period within intellectual development is reflected in higher order cognition or formal operational thought. In the years following the age of 12 , children learn to explore the world from multiple perspectives (Perrin \& Gerrity, 1981). Perspectives external to the adolescent can be considered in relation to a collation of logical outcomes, to determine alternative conclusions. This structural advancement in cognition permits children to hypothetically deduce logical inferences regarding illness causality.

The source and nature of illness continues to originate in internal physiological structures. Descriptions of illness extend beyond observable symptoms. Adolescents discern illness in relationship to internal bodily organs and functions, such as the heart and blood distribution (Koopman et al., 2004; Whaley, 2000). Often a failure or malfunction in internal physiological processes or organic biological structures is described. Children can conceptualize the necessity of interventions for specific medical complications. The removal of a previously functional biological structure resulting from poor functionality can be illustrated in pediatric patient's comprehension of tonsillitis. The development of health preservation and therapeutic interventions demonstrates adolescents' growing awareness of control over physiological states.

Children's conceptualization of the source, sequence, and period of recovery for illness progresses along a developmental continuum with comprehensive knowledge emerging with increased cognitive abilities and experience. Concepts of illness prevention and causation expand significantly through childhood. Such advances in health-related knowledge are predominately evident at the preoperational stage of development. With increased experience with illness, socially acquired standards regarding health and cognitive maturation, and drastic variations in the conceptualization of illness in the years of early childhood can be observed. For pediatric 
patients, accurate or erroneous representations of illness are determinative components in successful management of a medical setting.

\section{Enhanced Understanding of Illness Causality}

In the years following infancy up to adolescence, a child's appropriate interpretations of illness is a central component of their medical experience. For hospitalized pediatric patients, the ability to develop accurate knowledge regarding the origins, consequences, course, and treatment of illness can be valuable. Pediatric patients who demonstrate more sophisticated conceptual understanding of disease, perceive greater management of their healthcare setting (Koopman, et al., 2004). By developing an expansive understanding of illness, a stable framework to manage the emotionally demanding medical environment is accessible to young patients.

With development, children's representations of illness progresses from concrete discernible forces to sophisticated physiological mechanisms. This advancement in appropriate knowledge regarding the internalizing of physiological processes of illness can result in accurate concepts of prevention and therapeutic interventions (Bibace \& Walsh, 1980). For example, a pediatric patient who is aware of the biological mechanisms of illness will ingest medication as a compulsory act to benefit the internal organs within the body. With sophisticated understanding of internal processes, pediatric patients can more effectively comprehend procedures described by the medical staff. An advanced comprehension of physiological mechanisms can thus foster compliance within the medical environment. Pediatric patients with accurate representations of treatment more readily adhere to such medical procedures (Chilman-Blair, 2010; Eiser \& Havermans, 1992; Levetown, 2008; Perrin \& Gerrity, 1981; Whaley, 2000). Upon receiving an intervention, well-informed individuals can reflect on the necessity of a treatment. For pediatric 
patients, this can foster a sense of control over impending and possibly negative (e.g. painful) procedures and the medical environment (Koopman et al., 2004).

In addition, a pediatric patient with a comprehensive understanding of their medical diagnosis can discuss symptoms more clearly as related to internalized processes (Vatne, Slaughter, \& Ruland, 2010). Pediatric patients with an advanced understanding of illness possess a rich vocabulary of physiological structures and medical knowledge (Vatne et al., 2010). This can assist medical staff in treating prognoses more effectively. For example, an inexpert child may express the sensation of pain on his or her neck. However, the actual discomfort may be in the child's throat. A knowledgeable pediatric patient can promptly explain discomfort, seek relief, and develop accurate expectations for future medical experiences. The ability to effectively distinguish sensations and physiological structures allows a pediatric patient to advocate for their own health.

Health conscious pediatric patients can be an advocate for their own well-being and thus be encouraged to participate in their own medical experience. Pediatric patients with a suitable conceptualization of general concepts regarding illness, hospitalization, and health can be encouraged to actively engage in the medical environment (Levetown, 2008). By adequately acknowledging the extent of their condition, hospitalized children are permitted to make decisions regarding care (Rushforth, 1999). The practice of active involvement in medical settings can facilitate a sense of mastery and control within a limiting environment (Paterson et al., 1999).

A pediatric patient's perceived evaluation of control, and management over a medical encounter, has significant implications (Jaanister et al., 2007; Rushforth, 1999). Previous health management, or locus of control, can be defined as the extent to which an individual attributes 
medical outcomes to personal efforts or to chance (Shagena, Sandler, Perrin, 1988). An individual's locus of control is presumed to be directly related to health knowledge and understanding. Pediatric patients with more advanced conceptualization of illness express significantly more locus of control over their medical environment and outcomes (Koopman et al., 2004; Shagena et al., 1988). Children with an elevated locus of control can become selfassured in their intellectual understanding and management of illness (Chilman-Blair, 2010). These individuals with perceived control over their medical experiences possess greater knowledge regarding illness, treatment, and prevention, thereby promoting active participation in managing pediatric health.

The perception of control over one's overall medical experience can enhance a child's psychological well-being (Jaanister et al., 2007; Rushforth, 1999). Enhanced understanding of illness can facilitate effective management of arousal by modifying perceptions of control and the capacity to adjust arousal. A heightened sense of control in pediatric patients can thus enable greater exercise of effective regulatory strategies necessary to modify arousal (Jaanister et al., 2007). By modulating the intensity of arousal, a child's level of stress can be reduced.

Through developing an expansive understanding of the source, progression, and treatment of illness, pediatric patients can effectively manage the medical setting with ease. However, how a pediatric patient manages the emotional consequences of an experience is dependent on his or her subjective evaluation and appraisal of the emotional nature of the medical setting.

\section{Emotional Appraisal of Hospitalization}

An individual's interpretation of the relevance, consequences, and potential value of an event determines their subjective appraisal of an experience (Claar et al., 2002; Jaanister et al., 
2007). For a pediatric patient, the ability to navigate and manage a medical environment is governed by the child's evaluation of the medical setting. An individual's appraisal of the significance of an experience is initiated by recognizing and encoding perceptual features within the environment (Crick \& Dodge, 1994). By identifying and clarifying perceptual features of the experience, an individual can then interpret the significance of an event (Crick \& Dodge, 1994). Translating contextual cues into general appraisals involves a self-governed filtering process of identifying the risks and consequences representing an experience (Crick \& Dodge, 1994; Claar et al., 2002). In this initial appraisal of an event or setting, an individual attempts to identify physiological and psychological threats to their well-being.

Hospitalization can be emotionally threatening to young patients. The perception of threat is typically based on an individual's anticipation of personal harm (Lazarus, 1993). Other times, and quite often, the expression of anxiety, and distress regarding medical experiences, is emotionally discouraging (Claar et al., 2002). Unmet needs, a sense of danger, and a loss of control can be perceived as emotionally daunting to young patients (Lewick, 2013). The concept of illness itself can be perceived as intimidating to a hospitalized child. The transparent nature of an ailment and treatment options, can be difficult for a child to distinguish (Rokach, 2010). The distress experienced as a result of the illness and that of the treatment may become interchangeable to a pediatric patient. In addition, the severity of the illness, the physiological systems involved, treatments, and degree of discomfort experienced by the child, can represent realistic threats.

Perceived or realistic threats of an emotionally eliciting encounter can subsequently develop into negative emotions (Boekaerts \& Roder, 1999). Hospitalization can evoke an array of emotional reactions in pediatric patients. The emotions experienced by pediatric patients often 
reflect uncertainty and concern in their evaluation of the medical environment (Rokach, 2010). Recent work conducted by Bsiri-Moghaddam, Basiri-Moghaddam, Sadaghmoghaddam and Ahmadi (2011) has revealed a series of detrimental emotions expressed by pediatric patients in middle childhood. Children in the study identified feelings of fear, isolation, depressive mood, aggravation, and insecurity throughout hospitalization. The unknown surroundings and unfamiliar practices evoked suspense. Pediatric patients acknowledged additional distress regarding the fear of immobility, loss of limbs, and death. Participating children expressed anxiety related to pain provoked from diagnostic and treatment procedures. In addition, pediatric patients recognized elevated concern regarding separation from family members, friends and peers

The emotionally overt nature of hospitalization can be a hindrance or obstacle to the psychological well-being of a hospitalized child. How a pediatric patient appraises and manages possible emotional consequences to an experienced event develops as a secondary evaluation of the medical setting. A secondary interpretation of an emotionally eliciting experience is an individual's determination of available resources to manage emotions (Boekaerts \& Roder, 1999; Claar et al., 2002). An individual must initially select a preferred outcome by determining potential consequences for performing a response. Multiple responses can then be conceptually examined to predict possible directions to an event (Crick \& Dodge, 1994). In the self-interest of the individual, the most constructive reaction to an experience is then selected. Children's interpretations of contextual qualities of an experience, and their aptitude to direct preferred situational outcomes influence the meaning attributed to the situation (Crick \& Dodge, 1994). The development of secondary assessments encourages an individual to evaluate how the initial appraisal and arousal to the emotional event can be modified and controlled. 


\section{The Development of Emotions}

Emotions are naturally instinctive states of mind deriving from the environment, circumstances, or an individual's temporary disposition (Vohs \& Baumeister, 2011). The experience of emotions is biologically motivated resulting in physiological changes. These internalized reactions to emotional states produce bodily responses including fluctuations in the cardiovascular, cortical, and neuroendocrine systems (Cole, Martin \& Dennis, 2004).

Recognizable physiological responses include irregularity in heart rate and vagal tone in response to emotional provocation (Garber \& Dodge, 1991). In addition, observable displays of emotionality are depicted in the emotional cues of sensory states. Indicators of emotional arousal include the dilation of the pupils, as well as the constriction of major arteries and vessels within the body (Walden \& Smith, 1997).

Emotional states serve as signals to stimulate reactions and behaviors in response to relevant conditions within the environment. More superficial cues, such as distinctive facial expressions, allude to particular emotional conditions (Kopp, 1989). These expressions, such as a grimace, are visual signals. In addition, auditory responses can be exhibited to demonstrate the sensation of affective states. For example, a pediatric patient's response to the emotional state of fear may result in an audible response (e.g. cry, scream.

The expressiveness of affective states reflects an individual's personal reactivity to emotional encounters. Physiological reactions to emotional states are determined by an individual's predisposed response to emotional provocation, or emotionality (Blair, 2002; Kopp, 1989). An individual's perceived emotional reaction to a stimulus can determine the direction of the situational outcome. A highly emotional individual is temperamentally changeable, demonstrating negative valence and intense levels of emotional reactivity (Blair, 2002). 
Emotionally vulnerable pediatric patients often exhibit aversion to a situation. In contrast, an individual with low emotionality is temperamentally stable, demonstrates positive valance, and has modest levels of emotional arousal (Blair, 2002). A pediatric patient with low emotionality generally will approach the stimulus with confidence. The variability in the emotionality demonstrates the personalized nature of the experience of affective states.

To elicit the expression of emotions, an initial prompt must provoke a sensory response. For pediatric patients, an affective state can be promoted by encountering an emotionally eliciting experience. Emotionally eliciting experiences are an individual's appraisal of an encounter that elicits the expression of emotions (Thompson, 1991). An emotionally eliciting event can activate an assortment of emotional states. For example, the admittance into a medical setting may prompt sensations of fear, excitement, or sadness for a pediatric patient.

To delineate the significance of the event, children must first recognize, define and interpret the experienced emotion (Kopp, 1989). To effectively appreciate the complexity of emotions, children must be proficient in recognizing and labeling their own, as well as others' emotional expressions (Vohs \& Baumeister, 2011). Accurate knowledge regarding emotions permits children to internalize emotional experiences and respond correctly.

The identification, interpretation, and reactivity to emotional encounters is a subjective experience. An individual's judgment of an affective state is determined by evaluating the contextual features of the emotionally eliciting stimulus, as well as available resources to manage arousal. The ability to appraise a situation rapidly and promptly react to sustain favorable outcomes is an intrinsic property of emotional regulation. 


\section{Emotional Regulation}

To adequately adjust within an expressive environment, an individual must develop skills to modulate levels of emotional arousal. Emotion regulation involves the moderation of emotional responses through regulatory processes (Denham, 1998). Regulatory techniques are modification strategies associated with an activated emotional state. This management of emotions supports an individual's ability to maintain or modulate levels of arousal. To sufficiently respond to arousal, adaptable measures must be employed. Emotion regulation requires modification of emotional states to promote adaptive responses (Fox, 1994). An individual's capacity to respond to the explicit demands of an experience with a range of operational processes is a central component of human development. Emotional regulation is responsible for monitoring, appraising, and modifying experienced emotions through emotional, cognitive, and behavioral strategies.

Emotional regulation is valued as an instrumental tool in appraising experiences and dictating outcomes. This regulatory system is characterized by an individual's intended capacity to adjust to heightened levels of positive and negative emotions (Kopp, 1989). To successfully modulate affective states, an individual can generate or sustain an emotion to fulfill goals. An individual may down-regulate or reduce aversive or excitatory states (Gyurak, Goodkind, Framer, Miller \& Levenson, 2012). A constructive emotion may then be activated or upregulated to compensate for negative feeling states (Gyurak et al., 2012). This may also include a spontaneous or delayed reaction to emotionally eliciting situations to facilitate a favorable outcome. These processes of emotional control, such as expressive responses produced or inhibited in reaction to an emotional situation, are effective strategies exercised in the management of reactive and regulatory processes of affect. 
To accommodate the demands of the environment, children must acquire appropriate techniques to manage sensory information. Regulation of emotions requires individuals to alter the way in which they experience, perceive, and demonstrate emotions in order to accomplish short or long-term interests (Vohs \& Baumesiter, 2011). The adjustment of emotions to appropriate social responses by selecting applicable strategies is a developmental progression of available resources and optimal practices.

\section{The Developmental Trends in Emotional Regulation}

Emotional regulatory systems are developmental; in that they emerge and progress at varying stages throughout an individual's life (Garber \& Dodge, 1991; Kopp, 1989). The techniques children develop and use to monitor emotions change over time. These regulatory capacities emerge gradually, from external to internal means, to modulate emotions. Emotions are innately regulated in infancy as a means to monitor effective states (Cole et al., 2004). Over time, as social skills regarding emotional control are internalized, consistent strategies are acquired. Regulatory processes are developed and reconstructed though self-generated discovery (Kopp, 1989). Preliminary techniques to modify affective states are substituted for functional strategies. The ability to adjust the intensity and duration of emotions to the demands of a situation is a significant objective in emotional development.

Children under the age of two initially rely on behavioral approaches in monitoring experienced emotions (Denham, 1998). In early development, infants frequently recruit behavioral mechanisms to relinquish undesirable emotions. Reflexive patterns of behavior, such as primary circular reactions to manage external information, are activated in infancy (Kopp, 1989). Physical movements, such as repositioning of the head and body, can alter visible stimuli. Prior to the age of two, pediatric patients may resort to behaviors such as the closing of 
one's eyes, averting one's view, and gaze aversion to manage expressive states. Sucking of the hands and fingers, an instinctual reflex, can also be an instrument for self-regulation in infants. Physical movements of rocking and rubbing of the body are additional demonstrations of elementary self-soothing mechanisms (Kopp, 1989). Often, proximity to a secure adult to alleviate negative emotions is most frequently employed.

In infancy through the years of early childhood, the presence of a caregiver is beneficial to the development of emotional competence (Kopp, 1989). Regulation is initially provided by the child's external environment. The physical presence of a caregiver can provide direct means to diminish distress. Orientation, the positioning of a caregiver or attachment figure proximate to the child, can facilitate the development of self-regulation in children as a supportive technique (Rothbart, Sheese, Rueda \& Posner, 2011). Caregivers can demonstrate this technique by offering their hand as a source of comfort or by remaining present within an operating room throughout a medical procedure. The social interactions provided by caregivers and experienced peers helps children adjust, distinguish, and identify various feeling states.

Caregivers offer selective reinforcement, modeling practices, and optimal learning opportunities for the development of regulatory skills (Thompson, 1991). Effective caregivers assume roles as emotional moderators and facilitators. For example, caregivers can provide motivation for the development of self-regulation through instruction. A caregiver may suggest the child to close his or her eyes in the presence of a needle or for the duration of a medical procedure. In addition, self-regulation of emotions requires the development of behavioral schemes to alter or moderate amplified levels of arousal that are determined to be distressing to the individual (Kopp, 1989). These adult figures model sociably acceptable expressions of emotions. The process of conditioning reinforces the association between initially interpersonal 
events and regulatory functions (Kopp, 1989). As children successfully acquire regulatory skills, caregiver participation is reduced (Walden \& Smith, 1997). Physical self-soothing techniques diminish in early childhood with the emergence of more sophisticated, self-directed initiatives.

In the absence of caregivers, children begin to internalize cognitive processes during early childhood (Leerkes, Paradise, O'Brien, Calkins \& Lange, 2008). With the entrance into an educational setting and increased exposure to social institutions following the years of preschool, children are distanced from their primary attachment figures. Children must effectively extract formally employed regulatory skills from internal mental representations to develop selfmotivated cognitive mechanisms to modulate emotional encounters. Cognitive regulatory processes are self-guided methods to manage an emotionally eliciting stimulus (Denham, 1998). A child may redeploy attention externally by directing, preserving, or obstructing attention in an effort to modify levels of arousal. For example, a pediatric patient may divert attention away from an intravenous treatment to manage the experience of arousal. A child may also internally manipulate cognitive representations to reconstruct the meanings attached to a stimulus (Denham, 1998). Regulatory processes become more intrapersonal with developmental achievements in linguistic abilities appearing in the second and fifth years of life.

The development of language and communication in early childhood offers adaptive opportunities to regulate emotions. Increased proficiency in language in preschool provides children with a powerful tool for identifying, understanding, and communicating emotions (Kopp, 1989). The skills developed to effectively moderate expressed emotions are significantly improved in preschool. As a child acknowledges the feelings experienced, he or she is able to manage the situation in perceptual ways. For children, vocalization is a temporary means to reduce the experience of intense emotions (Kopp, 1989). Verbal descriptors of emotions can 
assist in the organization and reflection of expressive experiences (Vohs \& Baumesiter, 2011). The acquisition of language enhances children's conscious understanding and verbal expression of emotions. This developmental progression facilitates a range of abilities to regulate emotions in childhood (Cole et al., 2004).

As children become proficiently mobile, the available environment and opportunities to interact in social settings expand. This upsurge in accessibility has additional implications in the development of regulatory processes through socialization. Entrance into preschool or a primary educational setting facilitates the development of societal expectations regarding expressiveness. The ability to regulate emotions and produce acceptable behaviors is a major developmental task in early childhood (Fox, 1994). Display rules are societal conventions that determine where, when, and how emotion related behaviors are expressed (Carlson \& Wang, 2007). With age, children become increasingly aware of cultural guidelines regarding the presentation of emotions. In grade-school, children expand their social knowledge (Thompson, 1991). Children develop a comprehensive understanding of societal expectations regarding the expression of emotionality (Kopp, 1989). Conforming to emotional display rules becomes more evident in middle childhood.

As children develop, behavioral and cognitive responses are substituted for more sophisticated expressive regulation strategies (Denham, 1998). Social strategies diminish with the increase of self-guided initiatives and cognitive maturation. Children habitually construct autodidactic strategies to alter the range of emotions experienced. Expressive regulatory strategies attempt to alter the emotional intensity of arousal by minimizing or amplifying emotional states. An individual can adjust the strength, augmentation, threshold, and potentiality of the expressed emotion to contribute to a situational outcome (Denham, 1998). To successfully 
modulate arousal, an individual can inhibit, generate, or sustain an emotion to fulfill goals. A positive emotional state may be activated and maintained by a pediatric patient to counteract a negative encounter (Gyurak et al., 2012). In contrast, an individual may down-regulate or diminish an aversive state to facilitate a desired outcome (Gyurak et al., 2012).

Processes of emotional control, such as, behavioral, cognitive, and expressive responses, enhance children's capacity to moderate states of arousal. The capacity to develop and implement effective strategies of emotion regulation can assist in the management of emotionally eliciting encounters. By modifying affective states, children successfully direct behavioral outcomes. However, applications of regulatory processes of affect develop in progression with cognitive resources.

\section{Cognitive Implications of Emotional Development}

The development of regulatory abilities in childhood emerges gradually. Researchers suggested that this continuous progression of regulatory attributes is intrinsically associated with the development of cognition (Bell \& Wolfe, 2004). Emotion and cognition are dynamically connected and collaborative, organizing information into explicit behaviors and reactions. Characteristics of cognition that facilitate the management of information, maintain attention, and create problem-focused solutions, are synonymous with the regulation of emotion (Vohs \& Baumeister, 2011).

Cognitive control processes, which are comprised of a collection of techniques, are often identified as executive functioning. Executive functioning requires a set of cognitive resources to direct anticipated behavioral outcomes. An individual's ability to deliberately control thoughts and actions is a primary task of executive functioning (Carlson \& Wang, 2007). These processes work as a cohesive system to consolidate complex material and regulate thought and behaviors in 
an objective manner. In the process of organization and assimilation of information, executive functions include inhibitory control, shifting of attention, working memory, and planning (Vohs \& Baumesiter, 2011).

Planning. The successful integration of cognitive and emotional process begins to develop in the preschool period (Rothbart et al., 2011). This complex progression is initially exhibited in the ability to plan in early childhood. Planful cognitive activities are described as mental representations of outcomes, expectations of solutions and management of behaviors in order to accomplish a goal (Kopp, 1989). Cognitive strategies to plan, are valuable resources in the regulation of emotions. Perceptual plans of emotional regulation involve identifying, interpreting, and appraising contextual representations of the cause of distress in determining a sequence of strategies to modify an experience (Crick \& Dodge, 1994; Kopp, 1989). For example, an individual may anticipate solutions to ease anxiety regarding the perceived outcomes of an aversive experience (Kopp, 1989). Although a central component of cognitive processes, the ability to anticipate situational outcomes would not be effective if not for the capacities of working memory.

Working memory. Working memory is a set of flexible cognitive processes that enables preservation and manipulation of information from short-term memory to facilitate a goal (Gyurak et al., 2012). The function of working memory is to enable proficient conservation and applicable use of information over brief periods of time (Vohs \& Baumesiter, 2011). This process of monitoring and providing systematic feedback requires cognitive management of sensory experiences. Central components of the multifaceted cognition process of working memory involve the central executive and three substantial storage subsystems. 
The multidimensional, cognitive system of working memory is managed by the central executive. The central executive is a collection of cognitive processes that permits flexible processing of information to achieve a purposeful task (Vohs \& Baumesiter, 2011). The ability to complete a deliberate cognitive task requires the aptitude to preserve significant material while managing additional information (Schmeichel, Volokhov, \& Demaree, 2008). The central executive system is responsible for such cognitive control. The distribution of attention to goal oriented information by suppressing irrelevant responses that interfere with an objective is a task completed by the central executive. In addition, the central executive facilitates the aptitude to shift between tasks to enable flexible manipulation and updating of context relevant material. A final component of working memory is a set of three storage subsystems. The phonological loop, the visuospatial sketchpad, and the episodic buffer are responsible for combining complex cognitive information into significant reappraisals of relevant material (Vohs \& Baumesiter, 2011). The phonological loop retains verbal and acoustic information. The phonological cognitive system can permit an individual to disregard unattended or detrimental verbal messages. The visuospatial sketchpad manages visual and spatial information (Vohs \& Baumesiter, 2011). For example, a pediatric patient can control their visual attention by altering their gaze away from a salient visual stimulus or medical encounter. A final storage system, the episodic buffer, is the interface between long-term memory and the central executive (Vohs \& Baumesiter, 2011). The purpose of the episodic buffer is to integrate a range of information concerning experiences, or episodes, into long-term memory to allow for recall to resolve future tasks. Cognitive representations necessary for problem solving are developed as part of the episodic buffer (Baddeley, 2000). For example, the episodic buffer can develop into schemes or mental representations for pediatric patients regarding prior medical experiences. An individual 
can adjust behaviors and emotional responses by comparing actual experiences with predicted schematic expectations (Case et al., 2005).

The primary components of the central executive and three storage sub-systems of working memory have significant implications for the regulation of emotions. The manipulation, preservation and augmentation of emotional experiences are promoted by working memory (Vohs \& Baumesiter, 2011). Working memory encourages the regulation of emotions by facilitating storage and manipulating of perceptual and contextual information. Accurate representations of goal relevant information to manage emotional eliciting experiences are required (Vohs \& Baumesiter, 2011). This flexible attempt of processing information and sensory experiences results in voluntary regulation of the expression of emotion by inhibiting undesirable thoughts and emotional reactions (Schmeichel et al., 2008). For example, a pediatric patient may suppress facial expressions of negative emotions for the purpose of a desirable outcome.

In addition, cognitive mechanisms develop in congruence with working memory and assist in shaping future emotional experiences. Working memory provides substantial capacities to manage and manipulate sensory information into purposeful assessments to direct the expression of emotions and goal-oriented behaviors (Baddeley, 2000). Through such cognitive control, individuals can generate methods to manage expectations and construct adaptive regulatory processes necessary to redirect emotionally eliciting experiences.

Executive attention. Executive attention, a second component of executive functioning, refers to the ability to flexibly shift cognitive focus by selecting relevant aspects of the environment (Vohs \& Baumeister, 2011). The capacity to attend to relevant material enables individuals to focus on goal oriented information and disregard irrelevant environmental 
inferences. The process of attention can be isolated into three correlating mechanisms: alerting, orienting and executive control. The first process, remaining alert, is achieving and preserving arousal to attend to external surroundings (Vohs \& Baumesiter, 2011). By remaining alert, an individual can concentrate on contextual features of the environment to understand the full range of an encounter. A second practice, orientating, is the selection and alignment of attention to sensory information (Vohs \& Baumesiter, 2011). An individual can direct attention to specific sensory features within the environment. To accomplish this task, an individual can avert attention away from one stimulus and transfer attention to a separate stimulus.

The final considering factor is the role of executive control in the management of attention. Executive control is responsible for modulating, redirecting, and resolving conflict regarding an individual's perceptions, emotional states and behavioral outcomes (Rothbart et al., 2011; Vohs \& Baumesiter, 2011). This system shapes an individual's ability to intentionally inhibit, initiate, or alter attention. Cognitive tasks of planning, detection of faulty logic and integration of relevant information to select voluntary behaviors are central components in the regulation of emotional states. Children high in executive control are more proficient in managing attention, emotions, and behavioral responses (Bell \& Wolf, 2004; Carlson \& Wang, 2007; Rothbart et al., 2011). As a result, these individuals rarely display negative emotional reactivity.

Children develop voluntary systems of attention that enables the use of effortful strategies to modify levels of concentration to meaningful perceptual cues (Leerkes et al., 2008). The ability to flexibly alter the focus of attention, or cognitive resources, to adjust behavioral outcomes is known as attentional shifting (Vohs \& Baumesiter, 2011). In the development of regulatory control, individuals learn to divert attention by engaging in a secondary task to limit 
the focus of an emotional stimulus. This can be done by redeploying attention through internal or external means. The experience of emotions can be regulated by shifting attention to less taxing aspect of the environment (Denham, 1998). An individual may internally alter their attention by refocusing their thoughts in a constructive manner. This redirection of actions, or deliberate alteration in the sequence of events, can relieve the experience of negative emotionality (Kopp, 1989).

The attentional processes of alerting, orienting, and executive control are essential to the modification of emotional reactivity (Rothbart et al., 2011). An individual's proficiency to develop attentional cognitive mechanisms contributes to attempts to cope actively with stress.

Inhibitory control. A final characteristic of executive functioning is inhibitory control. Inhibitory control is characterized as the capacity to repress salient mental representation, or behavioral response that is inconsequential to a particular occasion (Carlson \& Wang, 2007; Vohs \& Baumesiter, 2011). This constructive regulatory technique enables the suppression of undesirable emotional experiences through attentional control and repression of inappropriate emotional displays.

Inhibition of faulty cognitive representations requires the ability to redirect levels of attention to nonessential stimuli (Leerkes et al., 2008). This can be accomplished by disregarding a stimulus, restricting available information, or by separating one's self physically from the emotionally eliciting encounter. Inhibition of behavioral responses to emotional states requires an individual to suppress primary responses for constructive emotional displays. Inhibition of physical emotional displays expands considerably during the periods of preschool in early childhood (Carlson \& Wang, 2007). 
Preschool aged children, from the ages of three to five, often alter their emotional experiences by modifying the presentation of sensory displays. Entrance into educational settings can assist in the inhibition of a dominant response by activating a secondary, sociably acceptable response. Societal expectations regarding expressiveness, or display rules, develop in congruence with socialization. Knowledge of cultural guidelines regarding the presentation of emotions determines behavioral expressions of affective states (Carlson \& Wang, 2007). Mastery of inhibitory control is an instrumental resource to successfully respond to emotional arousal in sociably appropriate manners.

Cognitive mechanisms of executive functioning such as working memory, executive attention, and inhibitory control operate as a cohesive system in the organization of complex information and in the regulation of behaviors. An individual's capacity to cognitively focus, repress, and preserve contextually relevant material is intrinsically associated with the regulation of emotions. This collaborative relationship of emotionality and cognition can be demonstrated at a neurological level.

\section{Integration of Emotional and Cognitive Processes}

Complex interactions between segments of the brain that address emotional responsiveness and a variety of cognitive functions can be observed throughout development. Neural mechanisms underlying emotion regulation are suggested to correspond to underlying cognitive neurological processes (Blair, 2002; Gray, 2004; Leerkes et al., 2008). The structures of the anterior cingulate and the prefrontal cortex have been determined to be central components of neural cognitive-emotional processes.

The cortical region of the anterior cingulate cortex contributes to the application of cognitive and emotional operations responsible for preserving attention and modifying levels of 
arousal (Vohs \& Baumesiter, 2011). This neural network modulates emotions by maintaining and orientating sensory events. The cortical region of the anterior cingulate cortex facilitates selective attention to perceptual cues to identify and flexibly manage emotionally relevant material (Posner \& Rothbart, 2000). Regions of the anterior cingulate cortex enable an individual to attend to or deflect debilitating stimuli.

An additional cerebral structure, the prefrontal cortex, facilitates effortful control processes of inhibition (Carlson \& Wang, 2007; Fox \& Calkins, 2003). This neural network evaluates perceptual features of the environment to exert effortful control in the direction of approach or avoidant behavioral outcomes (Bell \& Wolfe, 2004; Fox \& Calkins, 2003). The prefrontal cortex can inhibit a maladaptive dominate response to enable a goal-directed response. An emotionally eliciting encounter can be resolved in a constructive manner as a result of cognitive components of the prefrontal cortex.

The integration of the neural structures related to emotion and cognition are actively interrelated (Gray, 2004). Processes of cognitive control and emotion can selectively influence each other. For example, emotions provide a modulatory preference to prioritize capacities of cognitive control over others to assist in resolving control dilemmas (Gray, 2004). Biological pathways of central cortical structures of cognition and emotionality facilitate adaptive selection and application of regulatory strategies. Neural regions of emotional expression and cognitive processes intersect, supporting the premise of an integrated relationship.

The experience and application of emotional states and cognition processes are often indistinguishable and mutually dependent components of human development. Emotional states serve as signals to motivate and organize affective responses and behaviors to relevant contextual features of events and the environment (Gray, 2004). By reacting in a systematic 
manner to emotionally eliciting encounters, deliberate cognitive mechanisms are employed to facilitate goal directed responses (Lemerise \& Arsenio, 2000). Coordinating efforts of cognitive processes and emotional states are integrative psychological functions to serve in the resolution and direction of preferred situational outcomes.

The intersecting mechanisms of cognitive and emotional control interact through a series of steps of an individual's processing of an event or environment. This process, originally devised by Crick and Dodge (1994), is a theoretical component of social information processing. Crick and Dodge's (1994) model of social information processing is concentrated on the direction of behavioral outcomes of children in accordance with their interpretation of an experience. The theory delves into children's analysis of perceptual cues within a social situation to arrive at optimal responses (Crick \& Dodge, 1994). This rapid cognitive appraisal operates by attending to, encoding, and interpreting signals to permit an individual to clarify goals and generate a series of reactions to select an anticipated outcome to an experience. In recent years, the model has been restructured by Lemerise and Arsenio (2000) to incorporate emotional processing. The subsequent section of this review of literature explores this theoretical contribution.

The expression of emotions allows an individual to adapt subtle cues into general appraisals of situations (Gray, 2004). This process is initially employed by selectively concentrating on situational and internal cues to determine the nature of the experience (Lemerise \& Arsenio, 2000). The preliminary emotions expressed upon entering an experience can have significant implications on the cues received by the individual. An individual's perceived level of arousal to an event can serve as internal emotional cues (Crick \& Dodge, 1994). For example, a child may avoid or disregard emotionally eliciting information in 
anticipation that it will increase his or her levels of arousal (Case et al., 2005). Sensory information managed or inhibited by the child can modulate levels of arousal by directing attention to pertinent, less emotionally eliciting material. This ability to observe and examine the conditional or emotionally relevant features of an event is a central aspect of cognitive processing (Gray, 2004).

By recognizing and encoding the perceptual features of the experience, an individual can then interpret the significance of an event (Crick \& Dodge, 1994). Interpretation involves a selfgoverned filtering process of appraisals. To alter the initial affective response, cognition is required to translate an individual's perceptional reaction of an experience into a general appraisal. An individual can observe contextual features, or cues, within an environment to generate an overall evaluation of the situation at hand. An individual's appraisal of an experience is influenced by recalling prior incidences of related encounters. Children develop an archive of prior representations of experiences, or cognitive schemes, to appraise relevant physical and emotional features of an environment.

Applying preexisting schemes as a guide, an individual can adjust behaviors and emotional responses by comparing actual experiences with predicted schematic expectations (Case et al., 2005). However, recall of emotionally eliciting events, as well as the emotional intensity of a prior encounter, can directly influence a child's interpretation of a current or even up-coming situation (Crick \& Dodge, 1994). For example, in the recall of previously painful medical encounters, a pediatric patient's interpretation of an unrelated upcoming procedure may be misrepresented.

Through schematic interpretations and the review of possible reactions to an event, individuals can develop methods to manage expectations and construct adaptive regulatory 
processes necessary to redirect emotionally eliciting events. To initiate the management of an experience, a preferred outcome to the situation must be defined (Crick \& Dodge, 1994). The outcome, or a preliminary goal, must be selected after identifying situational characteristics of an experience. A goal can be internal, such as the means to maintain or regulate emotions, or an external instrumental objective, such as a reward (Lemerise \& Arsenio, 2000). However, the emotional tone of an experience can modulate the cognitive processes involved in determining a goal. Emotions can strengthen or obstruct a child's motivation to respond to a goal (Crick \& Dodge, 1994). The sensation of anxiety, for example, can direct avoidant goals. In response to an emotionally eliciting situation, a pediatric patient's preferred goal may be to withdraw or separate from a medical encounter.

After a desired outcome is determined, potential reactions to a situation are developed and considered (Crick \& Dodge, 1994). By accessing cognitive memory systems, multiple responses can be conceptually examined. An individual assesses previously retrieved responses to generate plans for how to effectively respond. The ability to flexibly shift between different options and various perspectives to anticipate a response to an event are fundamental mechanisms of cognition (Case et al., 2005). The responses an individual mentally infers are directly influenced by the emotional potency of the experience (Lemerise \& Arsenio, 2000). For example, during events that elicit anger, pediatric patients are more likely to remember responses made during past events that made them angry.

After assessment of potential consequences for performing the response, the most constructive reaction to an event must then be selected in the self-interest of the individual (Crick and Dodge, 1994). Responses to an experience are often determined by an individual's evaluation of the appropriateness of the response (Crick \& Dodge, 1994). For example, the 
expression of emotional states can be regulated by perceived social conventions of display rules. An individual retains schematics of rules and social knowledge regarding the appropriateness of emotional expressiveness (Lemerise \& Arsenio, 2000). Societal guidelines related to the expression of emotions can direct an individual's intended outcome behaviors. A pediatric patient may restrain from demonstrating a primary emotional response to facilitate the presentation of a secondary reaction. This secondary emotional reaction may be the individual's preferred outcome and may also serve as an expected outcome for the individual by others (Crick \& Dodge, 1994).

Governing constructs of emotional expression and cognitive processes function as interpretive assessments of relevant information to direct behaviors (Lemerise \& Arsenio, 2000). These multifaceted processes operate on a cooperative feedback loop serving distinct functions to facilitate optimal individual outcomes (Vohs \& Baumesiter, 2011). Cognition and the experience and management of affective states can be coordinated towards efforts to influence solutions and responses. However, for hospitalized patients, the emotional eliciting nature of a medical environment can often disrupt effective cognitive and emotional regulatory processes.

\section{Dysregulation of Emotions and Cognition}

The contextual demands of the medical setting can impact an individual's ability to effectively employ psychological functions of cognition and emotional resources, to serve in determining and guiding preferred conditional outcomes. Specifically, in a hospital setting, pediatric patients are confounded with elevated levels of emotionally eliciting events (Spirito et al., 1994). Increased contact with stressful events may act as an intrusive deterrent in the individual or collaborative efforts of cognitive processes and emotional states. 
Over exposure to highly emotional circumstances can interfere with an individual's organization of cognitive and emotional systems (Fox, 1994). Acute anxiety during hospitalization can impair the functionality of cognitive and emotional regulatory capacities by competing for available resources (Eccleston, 1994). These individuals may become overwhelmed with the intensity of emotional arousal, thus impairing fundamental internal processes of cognition and emotional control. Individuals who experience persistent emotional arousal are often unable to evaluate a situation from varying cognitive and affective perspectives (Lemerise \& Arsenio, 2000). Emotionally strained pediatric patients most often develop disorganized cognitive and emotional processes (Garber \& Dodge, 1991).

Unsystematic cognitive processes have been revealed in the inaccurate conceptualization of illness by hospitalized children. Prior research concerning an individual's understanding of the source, progression and remedial treatment of illness, has revealed less sophisticated representations in hospitalized pediatric patients (Paterson et al., 1999; Perrin \& Gerrity, 1981; Shagena et al., 1988). Incomplete knowledge concerning the conceptualization of illness can exaggerate maladaptive thinking by restricting the processing of information (Lemerise \& Arsenio, 2000). Detrimental thoughts of guilt and shame regarding the development of an illness are often perceived by pediatric patients with inaccurate representations of health (Myant \& Williams, 2005).

The emotional management of illness as well as the conditional strains of the environment may interfere with pediatric patients' cognitive capacities to manage information, specifically in comprehending health-related material. For example, a pediatric patient may ignore medically relevant information in anticipation of eliciting a negative emotional response within themselves (Case et al., 2005). By disregarding pertinent information as a consequence of 
emotions, ambiguous representations of health and illness are often constructed and applied. Additionally, and in turn, inaccurate conceptualizations of illness can often produce perceptions of uncertainty leading to anxiety and distress among pediatric patients (Eiser \& Havermans, 1992; Koopman et al., 2004). As a result, children with disordered cognitive representations of illness often perceive poor self-control over their emotional states (Garber \& Dodge, 1991).

Perceived inadequate control over the expression and management of affective states can develop into dysregulated emotional processes. Dysregulation can be identified as an individual's ineffective control over the expression and regulation of emotions (Fox, 1994; Garber \& Dodge, 1991). These individuals are emotional vulnerable and often have difficulties in successfully employing flexible methods to adjust emotional states (Fox, 1994). Despite attempts to initiate adaptive techniques, dysregulated individuals often reproduce ineffective regulatory responses (Denham, 1998; Garber \& Dodge, 1991). Consequently, for pediatric patients, persistent use of futile regulatory strategies can reinforce maladaptive responses to emotionally eliciting events.

The inability to exercise effective cognitive and regulatory strategies can drastically modify a pediatric patient's medical experience. Hospitalization is a dynamic contextual experience, characterized by highly emotional circumstances. The increased complexity and psychological demands of the medical environment require flexible resources to manage emotionally eliciting experiences. Optimal cognitive and affective regulatory processes for pediatric patients can individually and in combination support successful adjustment to a medical environment. 


\section{Impact of Prior Hospitalization}

For young patients, a healthcare setting can be an emotional environment. In the United States, approximately six million children are hospitalized every year for varying conditions (Thompson, 2009).The stay away from home, limitations in mobility, physical symptoms, medical procedures, disturbances in sleep and lack of independence can all be demanding on children (Rokach, 2010). Very often, children from infancy to adolescence have a challenging time adjusting to this transition. Stressors specific to the diagnosis, the severity, and the individual perceptions of the nature of the illness can interfere with successful adjustment (Rokach, 2010).

How a pediatric patient negotiates a medical setting is reliant on how he or she comprehends illness, he or her preexisting emotion regulation capacities, as well as his or her adaption to emotions experienced during hospitalization, emotion adaption which itself can support or hinder understanding of illness. Both of these factors are dependent on the cognitive maturity of the child to comprehend illness and the child's emotion regulation skills can also be strongly influenced by a child's prior exposure to a medical setting (Redpath \& Rogers, 1984). Following increased experience with illness and frequent exposure to emotion-eliciting medical encounters, pediatric patients develop adaptive logic in understanding illness causality and in addressing emotions experienced during hospitalization.

Prior representations of experiences and previously determined capacities to manage encounters are readily available for children to direct regulatory resources. The recollection of emotionally eliciting encounters can strengthen modifiers of distress. Preexisting exhausted regulatory techniques can be recalled to reconstruct present and future experiences of emotions (Kopp, 1989). Once successful mechanisms for regulating emotions are established, these 
strategies are employed regularly with increased exposure. As an individual develops and practices mechanisms for managing emotionally eliciting situations, they become more skillful in adjusting effectively.

Children also establish more comprehensive definitions of illness with experience (Williams \& Binnie, 2002). Pediatric patients who have had reoccurring exposure to medical experiences, such as the chronically ill, have more detailed conceptual representations of illness in comparison to less experienced peers (Case et al., 2005; Crisps et al., 1996; Paterson et al., 1999). As a result of increased experience with illness, pediatric patients retain cognitive representations of the origins, consequences, courses, and treatments of illness. Utilizing previously determined representations of illness as a model, children are able to accumulate and transfer acquired knowledge into general definitions of illness (Crisps et al., 1996).With frequent exposure to medically related material and encounters, hospitalized children develop sophisticated conceptualization of personal health and illness.

Research pertaining to the implications of extensive exposure to medical settings has revealed conflicting findings. The present study strived to explore an accurate representation of the ramifications of increased exposure to hospitalization in pediatric patients in early childhood. In addition, the present research delved into limitations concerning constructs of illness conceptualization and regulatory processes of emotion in young pediatric patients. Recent literature has concentrated on detrimental features of medical settings, representations of health, and the management of emotionally eliciting encounters (Bsiri-Moghaddam, et al., 2011). However, such investigations have disregarded the integration of these constructs within a collaborative relationship. 


\section{Summary}

In summary, this chapter provides the reader with a background on the multidimensional relationship between emotional regulation and the conceptualization of illness in hospitalized pediatric patients. The ability to construct accurate representations of health, the development of flexible methods to redirect emotionally eliciting events, and available resources to manage a significant experience can enhance a child's medical experience. The developmental progression evident in early childhood facilitates a range of abilities necessary in successfully managing the hospitalization experience. Following the entrance into preschool, advances in language acquisition, social competence, and cognitive maturation facilitates enhanced representations of health and the development of adaptive techniques to adjust arousal. Therefore, the current study further explored the dynamic relationship between emotional regulation and the conceptualization of illness in the management of a medical environment for pediatric patients in early childhood. It was hypothesized that there would be a relationship between emotional regulation and the conceptualization of illness in pediatric patients from the ages of three to five. Pediatric patients with greater knowledge regarding the source, progression, and treatment of illness were expected to exercise enhanced mechanisms in managing emotional arousal through the course of hospitalization. The study also addressed the impact of hospitalization on the development of sophisticated representations of illness and regulatory processes of emotional states. Prior research alludes to the application of accurate cognitive representations of previously determined conceptualization of health-related knowledge with frequent exposure to a medical setting. It was therefore hypothesized that with increased exposure to a medical setting, more accurate explanations of illness as well as advanced strategies to regulate emotional arousal would be observed in pediatric patients. 


\section{Chapter III}

\section{Method}

\section{Participants}

Participants were registered pediatric patients in the Acute Care Unit at a local children's hospital in Morgantown, West Virginia. The Acute Care Unit services children and adolescents who require less intrusive life-supporting care. This medical environment services a range of pediatric concerns including hematological, oncological, and cardiac conditions. Less intrusive ailments such as, tonsillitis, diabetes, and skeletal fractures are also treated within this medical setting. In addition, a single caregiver of the selected pediatric patient was requested to participate in the investigation.

Participants for the study were comprised of 26 admitted pediatric patients in the Acute Care Unit at a local children's hospial in Morgantown, West Virginia. Of the requested caregivers of the associated pediatric patient, 26 individuals consented to participate in the investigation. As some families were proceeding to check out of the hospital, three requested caregivers declined to participate and to have their child participate in the present study. A power analysis indicated that 25 subjects were needed to have a power of .80 to detect a large effect size for the planned study analyses using a statistical significance criterion of .05 . The total study participants included $18(69 \%)$ males and $8(31 \%)$ female pediatric patients. Pediatric patients' ages ranged from three years approaching the age of six with an average age of approximately 4.75 years $(S D=.85$; see Table 1$)$. A total of $3(12 \%)$ of participants were three years old, 11 (42\%) were four years old, and 12 (46\%) were five years old.

Mothers represented 73\% $(n=19)$ of the caregiver's reports. The remaining demographic information and rating of pediatric patients' regulatory processes of emotions were completed by 
$4(15 \%)$ grandparents and $3(12 \%)$ fathers. Legal guardians and other representative adult figures were not identified as participating caregivers. The age of the caregiver population reflected an expansive range, from 25 to 56 years, with an average age of 34.5 years $(S D=8.30$; see Table 1$)$.

As identified by caregivers, children's extent of exposure to a medical setting was determined to reflect initial and extensive periods of hospitalization. Initial admittances were identified as a single visit to the hospital. In comparison, extensive periods of hospitalization entailed more than a single admittance or repeated visits to a medical facility. The extent of exposure to hospitalization ranged from a single admittance to 15 overnight stays. The average number of hospitalizations was 3.6 admittances $(S D=4.10$; see Table 1). Approximately equivalent representations of pediatric patients with initial and extensive periods of hospitalization were observed, with 54\% $(n=14)$ experiencing an initial admittance and 46\% ( $n$ $=12$ ) with previous overnight hospital admittances. Of pediatric patients with previous encounters with a hospital setting, multiple visits ranged from 2 to 15 admittances (see Figure 1).

\section{Procedures}

Data were gathered over two months in the Acute Care Unit at a local children's hospital in Morgantown, West Virginia. The research commenced following approval from the designated research location and West Virginia University Institutional Review Board. The Child Life staff at a local children's hospital disclosed children who were available for participation. A Child Life Specialist, employed at a local children's hospital, initially approached the primary caregivers of pediatric patients falling within the identified age restriction. Non-verbal pediatric patients were not approached.

The patient's age determined individual participation in the study. To effectively explore the developmental trajectory of the conceptualization of illness and emotional regulation, the age 
of the participants was restricted to three to five years of age. Additionally, efforts were made to recruit an equal number of pediatric patients who were experiencing an initial exposure to hospitalization and children who had prior exposure to hospitalization. The child's year of birth was required to correspond to the designated age restraint at the time of participation. The gender and ethnicity of the patient did not impede participation opportunities. The availability of ageappropriate participants took precedence over secondary demographic characteristics. The presumed medical condition of the patient was not a determining factor in participating in the current study because the conditions of health treated at the Acute Care Unit encompassed the interest for this study. Therefore, the location of the pediatric patient served as an identifiable medical characteristic of participants to ensure the protection of sensitive health-related material.

A parent or legal guardian was presented with a brief description of the academic thesis and was asked if the primary investigator could be invited to describe the research in greater detail. If identified as willing to receive additional information with verbal consent, the primary investigator approached the caregiver.

The research material was presented to caregivers and pediatric patients by the primary investigator. A verbal script was presented to caregivers identifying the purpose and procedures of the academic thesis. A separate verbal script was presented to pediatric patients attributed to limitations in linguistic and literary abilities at this developmental period. If both parties were identified as willing to participate, the primary caregiver was presented with the consenting document and the child was read the verbal assent script.

Following the primary caregiver's completion of consent forms and obtaining verbal assent from the child, the caregiver was presented with two documents: a Parental Participation Addendum and Parent or Guardian Consent Form. The Parental Participation Addendum 
described the parent or guardian rights and personal agreement to participate in the research investigation. In response to the Health Insurance Portability and Accountability Act, the Parent or Guardian Consent Form was designed to inform the caregiver of the medical risks and protective features for their child in participating in the study. The child was asked, with parent permission, to mark their thumb print or an $\mathrm{X}$ with a crayon as an identification of approval on the designated proof of assent document. To ensure the safety of the child, the ink used to complete this task was temporary and nontoxic. This process enabled a sense of personal ownership for the child as well as a creative process. If the participating child was unwilling, incapable, or was not permitted to mark a thumb print or an X, verbal assent was sufficient.

The caregiver was requested to identify general information regarding their child by the primary investigator. The General Demographic Questionnaire (Appendix A) was designed to be completed in five minutes. A second measure, The Behavior Rating Inventory of Executive Function-Preschool (Gioia, Espy, \& Isquith, 2003), was presented to the caregiver to rate daily behaviors of their child associated with regulatory, analytic and social functioning (Appendix B). The inventory was estimated to take approximately 10 to 15 minutes to complete. The researcher was available to clarify directions on the demographic or behavioral questionnaires. At the time of completion, the primary investigator verified that all statements were answered. Incomplete inventory questions were invited to be readdressed by the caregiver.

If multiple caregivers were present at the time of the investigation, the primary investigator further clarified the necessity for only one caregiver to complete the questionnaires, at which time the caregivers decided amongst themselves who would complete the forms. This individual was required to be the parent or legal guardian of the child. Neither gender nor age restricted the adult participant's capacity to contribute. Male and female caregivers were sought 
exclusively based on availability at the time of the study. The caregiver's relational status to the child (e.g. parent, grandparent, aunt) also did not impede on participation. The legal guardianship of the caregiver determined consent and involvement in the study.

The General Illness Concept Interview (Perrin, Sayer, \& Willett, 1990) was verbally presented to pediatric patients by the primary investigator (Appendix C). Additionally, the children were asked to draw a germ and verbally answer related questions using the protocol developed by Banks in 1990 (Appendix D). To ensure the physical and emotional safety of the child, the caregiver was permitted to be present during the interview. However, to avoid collecting inaccurate material, the caregiver was asked to refrain from initiating responses from their child regarding the questions. The pediatric participant was verbally presented with a series of eight informative questions. Each question was sequentially verbalized following an adequate period for the participating patient to reply. The researcher was permitted to repeat a question a second time if the child did not respond immediately. The researcher was permitted to reiterate the child's statement to clarify if the child did not understand the question and/or to prompt additional information.

Interviews were tape recorded, with permission from the parent or legal guardian. Each interview recording was assigned a numerical identification which served as an identifiable marker throughout the entirety of the study. The recorded interview was transcribed by the primary investigator. All identifiable markers verbally presented in the interview, such as the child's name and medically relevant material, were removed from the final transcript. Following the completion of the transcript, the original recording was destroyed. If the parent or legal guardian refused the use of the recording device, the primary investigator transcribed responses on a supplementary document. 
The final question of the measure required the child to use artistic materials, specifically crayons and paper, to create a drawing to complete the question. As required in medical safety procedures, each child received an identical, unopened package of crayons. The primary investigator offered to open or to allow the child to open the package, based on caregiver and child preference. The child was permitted to keep the material following the completion of the interview with caregiver permission. To prevent the experience of distress by the pediatric patient, a duplicate of the child's illustration was created by capturing a photograph of the image. The photograph of the child's illustration was taken using a University camera immediately following the completion of the questionnaire. The visual image did not include any identifiable features of the child or his or her location. The original depiction was offered to the child, with parent permission.

Participation was entirely voluntary. At any point in the investigation, the child and/or caregiver was permitted to decline or terminate participation. Refusal to participate did not impede on the patient's level of care. The protection of medical information and safety of the child was ensured. The names and research data of the children and their caregivers was kept private and confidential with identifying information removed and replaced with a study numerical identification marker. Only the primary researcher, research assistant, and faculty advisor had access to the research material, to protect the identities of participants. All data, research material and consent forms were stored in separate locked storage cabinets, in a locked office, at West Virginia University. Identifiable information was kept in a separate, locked cabinet from the de-identified participant data. All data collected and compiled into computer programs were stored on a password protected drive. 


\section{Measures}

Demographic information. Caregivers of pediatric patients were requested to disclose information such as the age and gender of the child in the General Demographic Questionnaire (Appendix A). The age of the child was determined by using the month, date, and year of birth. Caregivers were also asked to identify the child's frequency of exposure to hospitalization. Caregivers were requested to identify, other than the birth of the child, on how many occasions his or her child has been hospitalized overnight. Based on the response selected by the caregiver, the extent of exposure to a hospital setting was determined. In addition, the caregiver was requested to identify their relationship to the participating child as the mother, father, grandparent, legal guardian or other. If other was selected, the caregiver was asked to specify their response. A final question inquired on the caregiver's age.

Emotion control. The Behavior Rating Inventory of Executive Function-Preschool (BRIEF-P; Gioia, Espy, \& Isquith, 2003) is a clinical scale developed to evaluate behaviors of executive functioning of children in a range of social contexts (Appendix B). The scale is recommended for the complex nature of medical settings for preschoolers aged two to five years (Gioia, Espy, \& Isquith, 2003). To evaluate children's executive capacities, the scale is to be completed by a parent or guardian with frequent interaction with the child. The parent or guardian rates the frequency of the child's behaviors over the past six months using a three-point Likert Scale of never (1), sometimes (2), or often (3).

The inventory is divided into five subscales that are central components of executive functioning including emotional control, inhibition, shift, working memory, and planning; the first three subscales are used in this current study. The child's aptitude to moderate emotional arousal is addressed in the emotional control scale. Statements presented in the subscale include 
"Mood changes frequently" and "Has explosive, angry outburst." The inhibit scale assesses the inhibitory control processes of young children. Inhibition is an individual's ability to refrain from impulses at appropriate moments. Items associated with this measure include "Is Impulsive" and "Acts too wild or out of control." To measure children's flexibility in problem solving, the shift scale is used. This process describes children's ability to shift attention in accordance to the demand of the environment for a desired outcome. Examples of questions regarding shifting include "Is upset by a change in plan or routine" and "Becomes upset with a new situation." The working memory scale evaluates the capacity of the child to sustain attention and actively maintain cognitive information to complete a task. Samples of statements in the subscale include "Needs help from an adult to stay on task" and "Has a short attention span." The plan and organization scale entails dual mechanisms of executive functioning. The capacity to plan describes the development of strategies to complete future tasks. Behaviors justifying this mechanism include "Has trouble thinking of a different way to solve a problem" and "Does not complete a task even after given direction." The final component, organization, describes an individual's aptitude to arrange information to achieve a goal. Examples clarifying this executive feature are "Gets caught up in the small details of a task or situation and misses the main idea" and "When instructed to clean up, puts thing away in a disorganized, random way."

From the five subscales, three broader indexes of inhibitory self-control, flexibility, and emergent metacognition can be generated based on the measurement instructions and design. Derived from the inhibit and emotional control subscales, the inhibitory self-control index describes emotional and behavioral inhibitory control of the child. The Flexibility index, composed of the emotional control and shift subscales defines an individual's ability to modify attention and to modulate emotions and behaviors to complete a task. A concluding index, 
emergent metacognition, from working memory and plan/organization subscales, describes a child's ability to cognitively manage tasks by employing strategies to complete future oriented tasks. The inhibitory self-control and flexibility indexes were a particular focus of this study.

The individual scores of each subscale can be summed to represent a raw total score for that individual subscale. The raw scores of the subscales can be combined based on their corresponding index to calculate a raw total score for each index as well. A $T$-score value can then be calculated for each type of raw score for a comparison of the participating individual's score relative to the scores of a normative sample from the measurement developers for standardization based on the gender and age of child. Lower scores indicate greater degrees of functioning (Gioia, Espy, \& Isquith, 2003).

The Behavioral Rating Inventory of Executive Function-Preschool is an effective measure addressing domains of regulatory processes, analytic strategies, and social functioning that has strong reliability and validity. Internal consistency is high, with alpha coefficients ranging from .80 to .95 across subscales and indexes (Gioia, Espy, \& Isquith, 2003). The instrument is supported to be a consistent and stable measurement over time. Test-retest correlations across the subscales ranged from .78 to .90 with a mean of .86 and an average interval of stability of 4.5 weeks (Gioia, Espy, \& Isquith, 2003). Using a factor analysis, evidence of the accuracy of the measurement has been established by the original creators of the instrument (Gioia, Espy, \& Isquith, 2003). The measure possesses construct and concurrent validity. In comparing the scales within the inventory with independent measures assessing similar variables, convergent and divergent assessments of the validity of the measure, produces reputable results (Gioia, Espy, \& Isquith, 2003). Analysis of the inventory demonstrates the accuracy of the intended constructs. 
For the present study, internal consistency was high, with alpha coefficients ranging from .86 to .93 across the Behavioral Rating Inventory scales and indexes. Items within the inhibit scale were internally consistent (Cronbach's alpha $\alpha=.88$ ). Individual items within the shift scale were also internally consistent (Cronbach's alpha $\alpha=.86$ ). The final scale, emotional control, also possessed internally consistency (Cronbach's alpha $\alpha=.88$ ). Internal reliability for the inhibitory self-control index, comprised of items from the inhibit and emotional control subscales, was also present (Cronbach's alpha $\alpha=.92$ ). The final index at interest to the present study, the flexibility index, composed of the shift and emotional control subscale was internally consistent (Cronbach's alpha $\alpha=.93$ ).

For the current study, the $T$-scores of the emotional control, inhibition, and shift subscales and the corresponding inhibitory self-control and flexibility indexes were utilized. These subscales and indexes were selected to capture emotional control of modulating emotions and emotional behaviors among children in the age range of interest for this study, as well as, the measures use in hospitalized settings. The participating primary caregiver in this study was given a condensed version of the BRIEF-P. To reduce time for completion only the above-mentioned subscale and index items were presented.

Illness conceptualization. The General Illness Concept Interview (Perrin, Sayer, \& Willett, 1990) is a measure devised to detect variations in the developmental progression of illness causality in young children (Perrin, Sayer, \& Willett, 1990). The 8-question instrument provides a systematic assessment of pediatric patients' perceptions of illness (Appendix C). The instrument is developmentally appropriate to the linguistic abilities of preschool-aged children. Attributed to age-related limitations in literacy, an open dialogue and interview format is used. The measure is designed to be completed in 10 to 15 minutes contingent on the child's extent of 
clarity in responses. The concise nature of the interview permits the researcher to maintain the attention of the pediatric patient. In addition, the precise dialogue should not impede on the medical environment.

The structured interview questions request children to describe the experience of illness from an external perspective. Participating children report how an independent child can distinguish the sensation of illness. Methods of contamination are explored in questions with reference to the development and origins of illness. For example, a question regarding bumps on the skin, a common irritant experienced by children, further demonstrates children's pragmatic comprehension of illness. Inquiries concerning recovery and prevention are demonstrated in discussing concepts of avoiding illness, providing relief and the implications of medication.

Responses are organized by the inclusion of external agents, internal processes, and causal mechanisms for illness based on the measure design and instructions. Children's descriptions are also classified by the presence of multiple relationships not by the accuracy of the information (Perrin, Sayer, \& Willett, 1990). Children's dialogues are assigned to seven temporal categories according to the complexity of the response. A score of zero, Refused to Respond, is allocated to inability to respond or responses unrelated to the question. A score of one is assigned to ambiguous response (i.e. "I do not know"). Dialogue involving a phenomenon or a circular reaction is assumed to a value of two, Global Response. A circular reaction can be described as a chain of causal events leading to an ultimate outcome. Responses describing concrete external agents without justifications of internal bodily processes are classified into the third level of illness comprehension, Concrete Rules. A value of four, Internalization and Relativity, is assigned to responses recognizing the temporal natural of illness following the internalization of detrimental agents. Children's responses acknowledging a naïve interpretation 
of the interactions between the agent and bodily processes are grouped into the fifth category, Interaction. A final classification, Generalized Principles, is assigned a value of six, to responses identifying the internal mechanisms, causal processes and management of illness. A general representation of the understanding of illness causality is obtained by averaging each child's responses across all items. To explore children's conceptualization of illness causality, the average score of illness causality understanding will be utilized in the current study.

The items within the measure are reliable and internally consistent (Perrin, Sayer, \& Willett, 1990). Internal consistency is high, with alpha coefficients ranging from .85 to .94 across all questions (Perrin, Sayer, \& Willett, 1990). The instrument provides a conservative estimate of the developmental variations in the conceptualization of illness. For the present study, internal consistency was high across items (Cronbach's alpha $\alpha=.86$ ).

Ellen Banks (1990) also developed a structured interview as well as artistic component to assess children's understanding and conceptualization of illness (Appendix D). Children are asked to describe and illustrate a personal depiction of a germ. Each child is given an identical blank piece of paper and four prepackaged crayons. The visual representation provides a creative medium for demonstrating illness causality among preschool-aged children. The visual image depicting a germ is classified into distinct artistic variables established by Banks (1990). Patterns of lines with unrecognizable visual content are distinguished as scribbles. Representations of closed outlines with discernible eyes and mouths are identified as faces. The inclusion of faces, trunks and limbs are recognized as human forms. A final representational category, monsters, include illustrations of nonhuman features such as horns, spikes and predominant teeth. Each artistic classification was designed to allude to children's increased awareness of germs as living, unfavorable entities, and will subsequently be coded using ascending numeric values to allow for 
quantitative analysis. Participating pediatric patients' narratives, describing features of the image, were transcribed to ensure accurate interpretations and coding of the illustration. Banks (1990) also developed a corresponding interview question of "What is a germ?" to explore children's conceptual understanding of a germ. Categorical representation of descriptions will be organized into responses of inability to answer, explanations referring to the term bad, features describing dirt or dirty, characteristic of disease and other.

Interview transcripts as well as illustrations were coded by an undergraduate research assistant. The independent rater underwent training prior to the initiation of the study. During the practice period, the research assistant was presented sample interviews to code. To ensure the reliability of coding, individual items from the General Illness Concept Interview, Bank's (1999) interview subset question and illustration categories by the research assistant and the primary investigator were compared. Discrepancies in coding were discussed. Cohen's kappa was calculated prior to the collection of data to determine if there was strength in agreement between the two coders. There was agreement between the two coders, $\kappa=.623, p<.0005 ; \alpha=.83$. Upon determining inter-rater reliability, the research assistant continued onto the research material. Throughout the study, instrument drift checks were completed for 10 (26\%) of interviews and depictions to assure reliability. Cohen's $\kappa$ analyses revealed agreement between the two coders, $\kappa$ $=.814, p<.0005 ; \alpha=.98$.

For the current study, the germ drawing component and a subset of the original interview questions were used. A subset was used to avoid repetition with the General Illness Concept Interview and to create a shorter, more concise combined overall interview experience for the children. In addition, the enjoyable nature of the drawing question assisted in concluding the interview in a constructive manner. 


\section{Chapter IV}

Results

The calculated scores of participating children's illness conceptualization as measured by the General Illness Concept Interview reflected an overall average of $2.38(S D=.88)$ with a range of 0.00 to 3.43 for mean values. In congruence with the literature and based on the General Illness Concept Interview measure design, participants' scores were indicative of normative developmental competences in early childhood and reflected preoperational cognitive thought. Male pediatric patients' averages fell slightly below female participants' average scores on the General Illness Concept Interview, with a mean score of 2.1 $(S D=.88)$ for males and $2.94(S D=$ .32 ) for female respondents, $t(24),=-2.39, p=.03$. (see Table 2).

As designed by the measurements developers, The Behavioral Rating Inventory of Executive Function-Preschool scores can be standardized by age and gender using the measure's normative sample. The Behavioral Rating Inventory of Executive Functioning-Preschool subscale and index values are standardized by two age groups: two to three years of age and four to six years. Within the current study, two males and one female participant among the sample were under the age of four, falling into the lower age group. Male participants within this developmental niche attained average subscale values of 52.66 on emotional control, 56.33 on shift and 62.33 on inhibit (see Table 2). Such mean values represented the $59^{\text {th }}, 67^{\text {th }}$, and $88^{\text {th }}$ percentiles, respectively. Index means of 59.00 on flexibility and 62.66 on inhibitory selfcontrol were observed, ranking among the $81^{\text {st }}$ to the $85^{\text {th }}$ percentile. A single female participant, obtained averaged subscale values of 55.00 on shift, 59.00 on emotional control, and 66.00 on Inhibit (see Table 2). Mean values represented the $67^{\text {th }}, 76^{\text {th }}$ and $93^{\text {rd }}$ percentiles, respectively. 
Index means of 59.00 on flexibility and 64.00 on inhibitory self-control were observed, ranking among the $78^{\text {th }}$ to the $91^{\text {st }}$ percentiles.

The average inhibition scores of the remaining 22 participants, ages four to five years, was a mean of 54.26 reflecting the $63^{\text {rd }}$ percentile for males and 54.14 for females ranking within the $60^{\text {th }}$ percentile. Average shift scores demonstrated greater variability and statistical significance by gender, with a mean of 54.73 for males representing the $70^{\text {th }}$ percentile and the $46^{\text {th }}$ percentile for females with an average of $49.00, t(23)=1.08, p=.03$. As demonstrated in the mean scores of emotional control, male participants were positioned at the $80^{\text {th }}$ percentile with an average value of 61.20. Female participants, however, had again significantly lower scores with a mean score of 62.33 and $50^{\text {th }}$ percentile ranking for emotional control, $t(23)=1.55$, $\mathrm{p}=.03$. For those four approaching the age of six, index mean values demonstrated significant degrees of variance between genders. Within the $75^{\text {th }}$ percentile, male participants obtained a mean value of 57.53 on the inhibit self-control index. Females, in contrast, were positioned at the $59^{\text {th }}$ percentile, with an average score of $53.00, t(23)=1.15, p=.03$. Significant variability among mean values was also observed among gender within the flexibility index, with an average of 58.40 for males ranking among the $80^{\text {th }}$ percentile and a value of 49.71 for females representing the $48^{\text {th }}$ percentile, $t(23)=1.33, p=.02$. It should be noted that the variance among construct means and gender were significant except Inhibit; with boys possessing significantly higher scores on measures, indicating less aptitude on shift, control, inhibitory self-control and flexibility (see Table 2). Average Behavioral Rating Inventory of Executive FunctioningPreschool scores for both genders are displayed in Table 3.

Descriptive statistics of the Banks (1990) interview question "What is a germ" revealed a range of narratives categories (see Table 4). Descriptions of germs resulted in $15 \%$ of 
participants declining to respond or failing to define ("I don't know"), 30\% expressing explanations referring to the term bad ("They are bad and mean"), $8 \%$ featuring descriptors of dirt ("Germs are yucky stuff in your body"), 23\% referencing the internalizing of agents and disease ("They bite your belly and make you hurt") and 23\% of accounts falling beyond the specified categories ("You can't see it but they make food taste gross") developed by Banks (1990). In congruence with the methodology, participating children's examination of germs functioned as anecdotal information (see Table 4).

Accompanied by pediatric patients' narratives concerning the description of germs, the corresponding Banks (1990) interview illustration of a germ revealed little to no variance, with $69 \%(n=18)$ of children's depictions categorized as scribbles or patterns of lines (see Figure 2). The remaining $21 \%$ of depictions were distributed evenly across the other available categories. As a result in the lack of variance in the collected germ illustration, planned statistical analyses could not be conducted. Instead, participating pediatric patients' depictions served as anecdotal examples for further discussion (see Figure 3).

\section{Hypothesis 1}

The first hypothesis was that a relationship between emotional regulation skills and the conceptualization of illness in pediatric patients would be observed. To test this hypothesis, Pearson correlations were conducted examining the relationship between the General Illness Concept Interview and the Behavioral Rating Inventory of Executive Functioning-Preschool. The relationship between the Behavior Rating Inventory of Executive Function- Preschool-Tscore values of the inhibit, emotional control and shift subscales, and The General Illness Concept Interview average score were examined. In addition, the relationship between the inhibitory self-control and flexibility indexes $T$-score values of the Behavior Rating Inventory of 
Executive Function-Preschool and the General Illness Concept Interview average score were also explored.

The Pearson correlations revealed significant findings (see Table 5). The General Illness Concept Interview was significantly and negatively correlated with the Behavioral Rating Inventory of Executive Functioning-Preschool inhibit $(r=-.42, p=.02)$ and $\operatorname{shift}(r=-.35, p=$ .04) subscales with moderate to large effect sizes. As presented previously within the measures composition, the inhibit scale evaluates an individual's capacity to resist compulsive behaviors at appropriate moments, with higher scores indicating poorer abilities to refrain from impulses. Shift captures a child's skills in modifying attention to the demands of the environment for a preferred outcome, with higher scores revealing decreased flexibility in modifying attention. Therefore, as inhibition and shift scores increased, indicating less skill levels, General Illness Concept Interview scores decreased. In addition, results revealed that Emotional Control was not significantly correlated with General Illness Concept Interview $(r=-.32, p=.6)$. A child's aptitude to moderate emotional arousal was not related to general understanding of illness and health-related knowledge.

Furthermore, The General Illness Concept Interview was significantly and negatively correlated with the inhibitory self- control index $(r=-.45, p=.01)$ and the flexibility index ( $r=-$ $.37, p=.03$ ) with moderate to large effect sizes. As expressed within the structural design of the measure, the inhibitory self-control index, composed of the inhibit and emotional control subscales, referred to the emotional and behavioral inhibitory control of children. Higher scores on the inhibitory self-control index indicate insufficient competences. The Flexibility index, derived from the emotional control and shift subscales, defined a child's ability to modify attention, arousal and behaviors to complete a task, with higher scores also indicating lower skill 
levels in this area. Thus, as scores within the inhibitory self-control and flexibility Indexes increased, indicating insufficient aptitude, General Illness Concept Interview scores decreased.

\section{Hypothesis 2}

The second hypothesis was that for pediatric patients with increased exposure to a medical setting through overnight hospitalization, more accurate explanations of illness as well as advanced strategies to regulate emotional arousal, would be demonstrated in comparison to children with less exposure to hospitalization. Regression analyses were planned to examine changes in children's scores on the Behavioral Rating Inventory of Executive FunctioningPreschool and the General Illness Concept Interview by the amount of overnight admittances to a hospital setting. However, reports of hospitalization were not linearly distributed, but were representative of two distinct categorical variables: initial (54\%) and extensive exposure (46\%). An initial experience was determined to be a single overnight admittance to a medical facilitate. Extensive exposure was determined for pediatric patients hospitalized for two or more admittances. An independent sample $t$ test was thus performed comparing the mean values of The Behavioral Rating Inventory of Executive Functioning-Preschool subscales and indexes and General Illness Concept Interview averages by category of hospitalization exposure. The comparison groups of hospitalization exposure did not yield significant differences in General Illness Concept Interview scores or the Behavioral Rating Inventory of Executive FunctionPreschool Inhibition or Inhibitory Self-Control scores (see Table 6). However, the analyses revealed that the Behavioral Rating Inventory of Executive Functioning-Preschool scores of Shift, Control and Flexibility were significantly different based on hospitalization exposure (see Table 6). Individuals categorized as experiencing frequent encounters with hospital settings overnight $(M=59.75, S D=13.69, n=14)$ had significantly higher mean scores on subscales of 
Shift than children experiencing an initial exposure to hospitalization $(M=47.93, S D=5.10, n$ $=12), t(26)=-3.00 p=.01$. Significantly higher mean scores for Control were also found in individuals characterized as experiencing frequent hospitalization $(M=63.33, S D=13.41, n=$ 14) than children with an initial experience $(M=52.00, S D=10.32, n=12), t(26)=-2.43, \mathrm{p}=$ .02. A concluding finding was observed with significantly higher mean scores on the Flexibility Index for individuals categorized as experiencing frequent hospitalization $(M=63.75, S D=$ $15.30, n=14)$ than children with an initial encounter with a hospital setting $(M=49.64, S D=$ $8.12, n=12), t(26)=-3.00, \mathrm{p}=.01$. The analysis of group variance was not supportive of the hypothesis, as pediatric patients characterized as experiencing extensive periods of hospitalization attained significantly higher mean scores on subscales of shift, emotional control and flexibility, reflecting poor aptitude on the constructs of interest 


\section{Chapter V}

\section{Discussion}

The purpose of this academic thesis was to explore the complex, multidirectional relationship between emotional regulation and the conceptualization of illness in the management of a medical setting among three to five year olds. As preexisting research has neglected to investigate the constructs of illness conceptualization and regulatory systems of emotions as collaborating systems, the present study contributes to contemporary literature, expanding on the vulnerabilities in previous research. The study investigated the implications of hospitalization on pediatric patients' regulatory capacities of emotion and health-related knowledge as well as the interplay between these two skill areas. The comprehensive findings and respective hypotheses are further divulged in the subsequent sections.

\section{Hypothesis 1}

In congruence with previous research, the initial hypothesis explored the complimentary relationship between the regulatory processes of emotion and the conceptualization of illness in pediatric patients (Case et al., 2005; Crisps et al., 1996; Kopp, 1989; Paterson et al., 1999; Williams \& Binnie, 2002). It was hypothesized that pediatric patients with enhanced knowledge regarding the source, progression, and treatment of illness were expected to exhibit higher skill levels in managing emotional arousal. Novices in biological principles, remedial care and wellness guidelines were thus predicted to apply ineffective strategies to modify the experience of affective states. The analyses of informant measures and pediatric patients' narrative accounts revealed partial support for the considered hypothesis. The results determined that general illness conceptualization was significantly correlated with several constructs of emotional regulation, including attentional shift and inhibition and intersecting regulatory skills reflecting a 
combination of the two constructs. The statistical findings suggested that when such regulatory skill levels were proficient, competencies in children's general conceptualization were also observed. Congruent with the theoretical premise described by Piaget (1969) and the measurement, participating pediatric patient's conceptualization of illness were normative; reflexive of the preoperational stage of development, with accounts exceeding concrete cognitive processing. Conversely, when significant constructs of regulation including shift and inhibition or in combination with emotional control were perceived to be low (indicating less aptitude) general conceptualization of illness was subsequently less developed. Therefore, children's conceptualization of contagion, contamination, wellness guidelines and remedial care would be impaired.

As additional analyses demonstrated, the observation of elevated scores on the inhibitory self-control and flexibility indexes revealed to be related to insufficient competences of pediatric patients in regards to the conceptualization of health-related knowledge. Such findings feature a confounded conjecture. As observed within the structural design of the measure, the emotional control subscale directly contributes to the composition of the inhibitory self-control and flexibility indexes. Assessed exclusively, emotional control did not appear to contribute to the development of illness conceptualization within the study population. However, when collectively examined with the additional Behavioral Inventory Rating scales, emotional control is perhaps a significant contributor to the cognitive task of illness conceptualization.

As demonstrated in the study findings, the necessity of inhibition and attentional shift to manage the emotional complexity of an environment and free available cognitive resources to assimilate material are suggested, as observed in prior research (Crisps et al., 1996). The constructs of inhibition and shift, were in agreement with the present theoretical literature. As 
previously defined, inhibitory control requires an individual to suppress salient mental representations or compulsive behaviors at untimely occasions (Vohs \& Baumesiter, 2011). Shift, in distinction, describes a child's proficiency in modifying attention to the demands of the environment for a preferred outcome (Vohs \& Baumesiter, 2011). An individual's capacity to refrain from impulses and modify attention to the demands of an environment are constructive regulatory techniques reflexive to the management of affective states. This merited proposition supports Denham's (1998) evaluation of behavioral, cognitive and emotional efforts to modify arousal. An individual's proficiency to repress salient inferences to redirect arousal and to divert attention by engaging in a secondary task to limit focus on an emotional stimulus, mirrors strategies exhausted in emotional control (Denham, 1998). With limited abilities to suppress primary emotional responses and flexibly alter attention, emotional control is perhaps hindered. Subsequently, cognitive resources necessary for the conceptualization of health-related material are restricted.

The analyses are limited in that conclusions are not conducive to determining a causal relationship. The constructs of interest collectively represent an integrated model, with no explicit path. Compatible with previous research, it is conceivable to presume that the obstruction of a pediatric patient's capacity to manage health-related material, resulting in confounded representations of illness, can undermine an individual's perceived management of emotional states, producing perceptions of uncertainty, anxiety, and distress among pediatric patients (Eiser \& Havermans, 1992; Koopman et al., 2004; Myant \& Williams, 2005). A divergent inference is also probable. Rather, insufficient aptitude to develop and apply appropriate techniques to manage an emotional environment, such as a hospital setting, can strain an individual's proficiency in assimilating and accommodating sensory information 
(Garber \& Dodge, 1991). By disregarding pertinent information because of emotional arousal, ambiguous representations of health and illness are often constructed and applied. A final hypothetical model may also be deduced. As the study design did not permit causal relationships to be inferred, a collaborative and mutually dependent system operating together to determine outcomes, may be responsible for the statistical findings.

The research findings revealed the complexity of such intersecting constructs of emotional regulatory control and the processing of cognitive information. As demonstrated in the resulting conclusions, regulatory processes of emotions are not exclusively associated with illness conceptualization; rather in combination with regulatory processes of inhibition and attentional shifting, emotion control relates to the management of health-related knowledge among pediatric patients in medical settings. The findings convey the significance of investigating both the emotional and inhibitory and attentional shift skill levels in combination to more accurately examine how pediatric patients cognitive processing and needs may be influenced. Future research directives and interventions should focus on developing a comprehensive set of skills to help better care for children exposed to medical settings.

\section{Hypothesis 2}

A second hypothetical consideration was explored to investigate the conditional demands of a medical setting for those hospitalized overnight on a young child's capacity to effectively exercise functions of cognition and regulatory processes of emotion. In coherence with prior theoretical contributions, it was hypothesized that with frequent exposure to a medical setting overnight, more accurate explanations of illness and more advanced strategies to modify emotional arousal would be observed. Analyses were not supportive of the intended hypothesis. An independent samples $t$ test yielded significant results, with children characterized as 
experiencing frequent admittances to a medical setting exhibiting poorer aptitude of emotional control, shift, attentional control, and inhibition. These statistical findings contradict previous research (Case et al., 2005; Crisps et al., 1996; Paterson et al., 1999), suggesting that with increased experience with illness and exposure to emotion-eliciting medical settings, such as through hospitalization, more erroneous logic in understanding illness causality and increased application of ineffective regulatory techniques may occur.

As these constructs of interest have remained beyond the contemporary scope of recent literature, an alternative explanation may explain the divergent findings. That is, frequent exposure may not constitute implementation of effective regulatory strategies of emotion. Rather, persistent exposure to hospitalization for the child may be a recent occurrence. For example, a mother of a male pediatric patient expressed apprehension in disclosing the extent of his exposure to hospitalization within the past year. Medical intervention was recently sought, with no admittances prior to his diagnosis. Children more recently diagnosed with prognoses requiring immediate and continuous care may have limited experience implementing adaptive regulatory techniques effectively and consistently over time. Perhaps the present sample may represent a newly diagnosed population of pediatric patients. Moreover, it may not be that pediatric patients within the sample do not possess capacities to modify the experience of emotion arousal, but rather are inexperienced.

The current study findings are congruent with several research investigations previously conducted. Fox (1994) and Spirito and colleagues (1994) reported that disorganized cognitive and emotional processes may consequently be developed by emotionally strained pediatric patients. For chronically ill pediatric patients or children that require continuous medical care, extensive periods of hospitalization and elevated levels of emotional arousal in a medical setting 
may consequently hinder their capacity to relieve the experience of negative emotionality. Perhaps as levels of attention are directed to and concentrated on emotionally eliciting stimuli, adaptive cognitive and affective resources are restricted (Leerkes et al., 2008). Voluntary systems and effortful strategies to modify levels of concentration and inhibit less salient perceptual cues may become impaired, reinforcing faulty cognitive representation. Perhaps for the present sample, the emotional conditions of the medical environment obstructed the management of expressive responses, modification of attention and assimilation of pertinent material. As suggestive of the research findings paired with these previous works, it could be hypothesized in future investigations that children exposed to hospitalization for extensive recurring periods of time may become emotional strained, and in turn incite cognitive disorganization and weaken emotional process skills (Fox, 1994; Spirito et al., 1994). As a consequence of the emotional complexity of a medical environment, fundamental internal systems of cognition and emotional control subsequently become exhausted.

Although divergent from the intended research hypothesis, the study findings present a compressive depiction of vulnerabilities of pediatric patients chronically exposed to emotionally eliciting medical settings, such as through hospitalization. It is therefore pertinent for medical professionals and future interventions to readdress the protective care of hospitalized children to confront the emotional obstacles experienced by pediatric patients with extensive exposure to hospitalization. Such considerations are persuasive, as the present research contributes to emerging disciplines concentrated on addressing the vulnerabilities of pediatric patients requiring extensive, continuous medical care. 


\section{Limitations and Future Directions}

The present thesis investigation is instrumental in advancing literature on the resiliency and limitations of pediatric patients. In addition, the implications of the present study feature the necessity of advocating for the needs of pediatric patients and the manner to which support is implemented for this vulnerable medical population. Although the current study significantly contributes to contemporary literature, it is imperative to address limitations and future directions.

Child Characteristics. Given the exclusivity of participation, features identifiable to the sample population pose both strengths and limitations to the present study. First, variability among the participating pediatric patients' personalities may have substantially limited or skewed illness conceptualization accounts. For example, despite attempts to engage with the children prior to the presentation of questions, in the presence of an unfamiliar adult, such as the primary investigator, some wary children demonstrated apprehension in disclosing answers. Following the conclusion of the interview, several caregivers displayed grievances in their introverted child's accounts. For example, a mother of a participating pediatric patient expressed dismay over her child's suppressed responses. The female caregiver acknowledged that as a consequence of her child's reserved nature, concepts that were recently taught were omitted from the child's narrative.

In contrast, a number of participating children were intentionally uncooperative to elicit negative responses. For example, repetition of irrelevant answers or profanity, such as describing excrements, was uttered to provoke displays of disapproval. Such responses were disregarded or diverted to promote reengagement in the interview process. As demonstrated by a participating child's narrative, his initial response of "You're funny" to each inquiry was deflected and 
redirected by the primary investigator. Earlier irrelevant responses were then replaced with the child's actual answer regarding representations of illness and health-related knowledge.

Additionally, as a limitation of the current study, the linguistic constraints of participating children are of some concern. Vocabulary growth progresses from initial understanding of others' expressions to the verbal application of one's own thoughts and ideas. Young children often recognize and comprehend more words then they verbally produce (Bloom, 1998). Additionally, following the second year of life, children vary in the onset and development of word acquisition (Bloom, 1998). Specifically, at approximately two years, children can comprehend an average of 50 words; however, the production of words is limited to approximately ten verbal utterances (Bloom, 1998). As demonstrated in the utterances of the participating pediatric patients, verbal responses often lacked substances, connotation and precision. For example, a pediatric male participant's response to "What is a germ" possessed contradictory nuances: "Germs are good. When you see one, they hurt you."

Moreover, the implications of educational attainment for participating children's illness conceptualization should be addressed. Participating children frequently stated wellness guidelines, such as the washing of hands, guarding a sneeze, and maintaining a nutritious diet. Such narratives regarding standard health recommendations were accompanied by references to education. As confirmed in a pediatric patient's account of solutions to prevent illness, her knowledge was directed by and reinforced by her preschool teacher: "My teacher coughs on her arm. She says you have to wash your hands. I don't give germs because I wash my hands.” Children enrolled in educational programs may have increased exposure to health instruction and illness information. Entrance into compulsory elementary school or voluntary early education programs may influence conceptualization of illness and health-related knowledge. It is therefore 
recommended that researchers examine the educational experience of participants in the future studies in this area.

In addition, it is significant to recognize the variability in gender representations. The prevalence of pediatric male patients representing the sample is explained by and is congruent with national averages. In agreement with the Center of Disease Control and Prevention (2012), 5.4 thousand males under the age of 18 requested medical interventions for unintentional injuries, falls, motor vehicle accidents, wounds and intentional harm in 2010. Pediatric female patients by comparison accounted for 3.6 thousand yearly visits for medical services (CDC, 2012). As the gender composition of participants was not equally distributed in the study's sample population, future research should be directed to pursue a complete account of gender experiences with hospitalization.

Furthermore, it is relevant to appreciate the medical prognoses justifying the necessity of hospitalization. As the Acute Care Unit at a local children's hospital services a range of pediatric concerns, the intrusive nature of diagnostic and therapeutic procedures may have subsequently altered the cognitive and emotional resources of pediatric patients. Advanced congenital, autoimmune, and inoperable conditions including hematological, oncological, and cardiac disorders generally require extensive interventions and are psychosomatically taxing (Rokach, 2010). Features of the condition and responses to administered medication may perhaps obstruct normative functioning. For example, an interview with a young pediatric participant was completed independently from the caregiver questionnaires, as the administered medication produced drowsiness. Perhaps, low-grade medical ailments such as, tonsillitis, constipation and skeletal fractures are less obstructive to the functional capacities of pediatric patients. As the present investigation did not investigate or collect diagnostic information it would be applicable 
for future research to explore the implications of medical prognoses on pediatric patients' functions of cognition and regulatory processes of emotions.

It is also of particular importance to examine the present investigation's introduction of an unanticipated gender observation, specifically male participants' skill level. As reported within the analyses of descriptive statistics, the present male pediatric sample's skill levels in emotional control, shift, inhibition and in combination represented the $70-80^{\text {th }}$ percentiles compared to the measurement normative samples. Furthermore, the statistical findings are substantial, as pediatric female participant scores did not exceed standardized sample values. This finding is suggestive, as the experience of hospitalization may be especially detrimental to pediatric male patients. It would be substantial for future researchers conducting causal investigations to explore gender variability in constructs of emotional regulation in medical populations.

The child's age of participation, should be further noted. As a result of the control in participation, such as the restriction in pediatric patients' age of involvement, the findings are not generalizable beyond the age range of three to five years old. As the cognitive and regulatory competencies of three to five year olds is exclusive to their developmental period, conclusive accounts of those beyond the age of five cannot be inferred. As an asset of the current study, the age of interest served as a portal into the progression of regulatory processes of emotion and cognitive maturation, as it is an interim for intellectual, expressive and social development. The exploratory investigation served as an avenue to further address the growing needs and vulnerabilities of children within this critical developmental period who are exposed to hospitalization and illness. Future research should consider integrating children from various 
stages, in addition to those from the ages of three to five, to explore age variations in the response to hospitalization and illness.

Concluding considerations regarding sociological influences should also be investigated. The manner in which emotions are elicited and the meaning ascribed to emotional expectations are constructed within an individual's social context (Garner \& Spears, 2000). Traits of ethnicity and socioeconomic status offer evidence of variability in the development and enactment of emotions (Morelen, Jacobs, Suveg, Jones, \& Thomassin, 2012). As display rules are socialized as standard practices across cultures and vary according to ethnicity, such discrepancies across groups may apply. For example, as described in previous research, Asian American individuals report lower displays of negative emotions in comparison to Caucasian and African American counterparts (Morelen, et al., 2012). This ethnic distinction is further recommended by the researchers to be the result of cultural beliefs regarding familial disobedience and disrespect.

Furthermore, contrasting attitudes concerning the experience and expression of emotions varies across social classes. For example, encouraged expressions of anger in low-income and working-class neighborhoods may suggest personal qualities of toughness valued in such communities (Garner \& Spears, 2000). In addition, as demonstrated in relevant literature, emotional regulatory practices of seeking comfort from an adult, among low-income children, has been proposed to be a form of weakness and may result in mockery (Garner \& Spears, 2000). Verification of such divergent practices warrants clarity and further investigation.

A final conjecture, regarding parental emotional philosophies merits additional attention. Particular parental attitudes underlying the validity of emotional experiences, beliefs concerning the significance of attending to emotions, values concerning self-regulatory practices, and the connotation ascribed to controlling emotional displays directly contribute to the expressive 
outcomes of children (Meyer, Raikes, Virmani, Waters \& Thompson, 2014). Moreover, the expression of affective states is contextually bound. Thus emotional regulatory objectives of children as well as parental expectations are altered to match the emotionally eliciting encounter (Meyer, et al., 2014). Perhaps for hospitalized pediatric patients, normative regulatory processes of emotion are not presumed under the conditional restraints of a medical setting. For example, the unmanageable expression of anger or distress may be accepted by caregivers as an appropriate response to hospitalization. As the present study omitted such considerations, prospective research investigations should incorporated parental socialization practices and contextual expectations on the emotional adjustment of pediatric patients.

Methodological Considerations. The application of measures in the present academic investigation warrants further examination. Growing methodological contributions and constraints are addressed in the subsequent section.

The Behavior Rating Inventory of Executive Function-Preschool edition was a valuable tool in assessing the executive functioning of pediatric patients. However, the measure possesses inadequacies that warrant concern. The standardized instructions requests caregivers to identify how often each of the following behaviors has been a problem. The presentation of negative language within the measure's instructions may elicit thoughts of disapproving conduct among parents. Specifically, the direct emphasis on deviant behavior may have misguided caregivers to respond in a more favorable manner, as several parents within the present study expressed concern over the measure's clinical purpose of identifying developmental problems. To gather impartial accounts, amendments within the measure's presentation of instructions and design are merited. 
In addition, it is pertinent to examine the methodological restraints of Banks' (1990) classification of a germ. Regrettably, the measure minimized the extent of knowledge perceived to be salient in young children's descriptions of germs. A fifth and supplemental category was subsequently constructed to capture accounts of germs independent from the current stringent categories generated by Banks (1990). Several pediatric participating children provided detailed information relevant to characteristics of germs but exceeded the original categories constructed by Banks (1990). For example, descriptors of invisibility and tiny were frequently referenced. Additional accounts accurately described the presence of germs on extremities such as on the hands and fingers, as well as orifices, such as inside one's mouth and stomach. The extension on the proposed categorization of germ narratives yielded expansive knowledge regarding the physical properties, internal interactions and contributions to health (see Table 7). Future application of the presented interview question should be advised to utilize methodological principles of Grounded Theory. As a constant comparative method, Grounded Theory identifies reoccurring categories and patterns in participant narratives (LaRossa, 2005). By developing, revising and determining central themes through the narratives of participants, authentic accounts can be abstracted.

An additional methodological consideration regarding constraints in the illustrative depiction of a germ, utilizing Banks' (1990) method should also be contemplated. Although the visual representation was an enjoyable medium for participating pediatric patients to demonstrate their knowledge, the original measure underestimated the sophistication and imagination concealed within young children's imagery. As verified in research, the prevalence of illustrated patterns of lines and scribbles may be reflexive of developmental preferences. The tangle of lines illustrated in a child's depiction, as suggested by Winner (1986), may exceed meaningless 
doodles but obscure images manifested in gestures and imaginative representations. Scribbles, characterized by the moving of one's hands in a disorganized manner, may symbolize experimentation in imagery. As opposed to discernable pictorial forms, preschool-aged children apply gestural abstractions such as incorporating dialogue and movement to justify the content concealed within their depictions (Winner, 1986). To an ill-informed adult, a series of unsystematic lines may appear indecipherable. However, if requested to describe the image, children often label scenery, objects and human figures in great detail and certainty. In addition, the execution of scribbles requires large-scale movements that may imitate the direction and action of the intended character. For example, in the current study a participating pediatric patient scribbled vigorously a series of lines. When invited to describe the image, the child expressed that the rapid movements represented germs moving quickly throughout an individual's body.

Furthermore, the abstract physical characteristics of a germ may have challenged the cognitive resources available to participating children operating at the preoperational phase in development. Prior to onset of adolescence and formal operational thought, children falter to overlook concrete external inferences (Piaget \& Inhelder, 1969). Germs, which are perceived as undetectable microorganisms, were frequently referenced by participating children as invisible. The capacity to illustrate an undetectable object may have appeared to be an unfeasible task for those in the study's developmental period of interest.

The application of artistic mediums does offer a methodological contribution to future investigations. The presentation of artistic materials to express intellectual competencies in regards to the source, sequence and progression of illness causality is a prospective alternative to an exclusive question, "What does a germ look like?" As demonstrated by a four-year-old 
pediatric female participant, inquiries regarding the origin, temporal course and remedial care of illness were readdressed following the presentation of crayons and paper. The illustration encompasses the physical nature of germs, the internalization of the detrimental agent and the sequences of recovery (see Figure 4). As demonstrated in the female patient's depiction, a series of lines were produced to demonstrate the sequence of illness causality. The illustrated narrative begins with a number of circles representing germs in a large rectangle. The germs are subsequently internalized by a smiling, content individual portrayed in the center of the paper. Discontent that resulted from the development of an illness was then represented in a grimacing portrait of an individual. However, the ill individual received remedial care, as represented by the medication depicted as a square with a centered heart label and an individual resting in a bed. This depiction exemplifies the utility of artistic material as well as the intellectual capacities of young children in regard to illness conceptualization. This method permits children to better express the extent of their conceptualization of illness and health-related knowledge, inherently increasing the accuracy of the study data. It is suggested that future investigations into the conceptualization of illness with preschool-aged pediatric patients should apply artistic means through the entirety of the interview processes.

A final statistical limitation requires clarity. As the present study utilized minimal power criteria of .80 to detect moderate to large effective sizes with the current sample, the possibility of additional significant relationships, or even variations in findings, must be considered. It would be optimal for future investigations to amend the statistical power to detect smaller effect sizes by the addition of an increased sample size. 


\section{Conclusions}

For young patients, hospitalization can present children with a range of emotionally eliciting encounters and ambiguous medical endeavors. The demands of a medical setting can thus influence an individual's propensity to exercise functions of cognition and regulatory processes of emotion. This investigation established that regulatory dimensions of affect, comprising of inhibition, attentional shifting, and inhibitory self-control and the development of illness conceptualization, were cooperating constructs. In addition, extensive exposure to hospitalization was significantly related to impaired regulatory strategies of inhibition, attentional shifting, flexibility, and inhibitory self-control, incongruent with the literature (Case et al., 2005; Crisps et al., 1996; Paterson et al., 1999), although consistent with a few previous research investigations (Fox, 1994; Spirito et al., 1994). Although unforeseen findings, final considerations are pertinent to contemporary literature as the present study challenges former limitations by exploring cohesive systems of cognition and emotional adaption, operating collectively. In addition, this investigation validates the necessity of respective therapeutic models to appropriately intervene for vulnerable, medically fragile populations. As suggestive of prior literature, the need for emotional support for this vulnerable population was not predicted; however, the study has helped identify a group of young patients requiring additional emotional support. To better understand the emotional and cognitive needs of pediatric patients, exploratory consideration to disentangle the enmeshed experiences of these children should continue to be investigated in future research to promote adaptive outcomes to hospitalization at this critical period of development. 


\section{References}

Banks, E. (1990). Concepts of Health and Sickness of Preschool and School-Aged Children. Children's Health Care, 19(1), 43 - 48.

Baddeley, A. (2000). The episodic buffer: A new component of working memory. Trends in Cognitive Sciences, 4(11), 417-423.

Bell, M. \& Wolfe, C. (2004). Emotion and cognition: An intricately bound development process. Child Development, 75(2), 366-370.

Bibace, R. \& Walsh, M. (1980). Development of children's concepts of illness. Pediatrics, 66(6), 912-917.

Blair, C. (2002). Integrating cognition and emotion in a neurobiological conceptualization of children's functioning at school entry. American Psychologist, 57(2), 111-127.

Bloom, L. (1998). Language acquisition in its developmental context. In D. Williams (Ed.), Handbook of child psychology: Volume 2: Cognitive, perceptions and language (pp. 309370). Hoboken, NY: John Willey \& Sons Inc.

Boekaerts, M. \& Roder, I. (1999). Stress, coping and adjustment in children with a chronic disease: A review of literature. Disability and Rehabilitation, 21(7), 311-337.

Borzekowski, D. (2009). Considering children and health literacy: A theoretical approach. Pediatrics, 124(3), S282- S288.

Bsiri-Moghaddam, K., Basiri-Moghaddam, M., Sadaghmoghaddam, L., \& Ahmadi, F. (2011). The concept of hospitalization of children from the view point of parents and children. Iranian Journal of Pediatric, 21(2), 201-208.

Carlson, S., \& Wang, T. (2007). Inhibitory control and emotion regulation in preschool children. Cognitive Development, 22, 489-510. 
Case, A., Fertig, A., \& Paxson, C. (2005). The lasting impact of childhood health and circumstances. Journal of Health Economics, 25, 365-389.

Chilman-Blair, K. (2010). Communicating with children about illness. Practical Nursing, 21(12), 631-633.

Claar, R., Walker, L., \& Smith, C. (2002). The influence of appraisals in understanding children's experiences with medical procedures. Journal of Pediatric Psychology, 27(7), $553-565$.

Cole, P., Martin, S., \& Dennis, T. (2004). Emotion regulation as a scientific construct: Methodological challenge and directions for child development research. Child Development, 75(2), 317-333.

Cowen, P. (1979). Piaget: with feeling: Cognitive, social and emotional dimensions. New York: NY US: Holt, Rinehart and Winston.

Crick, N. \& Dodge, K. (1994). A review and reformulation of social information-processing mechanisms in children's social adjustment. Psychological Bulletin, 115(1), 74-101.

Crisps, J., Ungerer, J., \& Goodnow, J. (1996). The impact of experience on children's understanding of illness. Journal of Pediatric Psychology, 21(1), 57-72.

Denham, S. (1998). Emotional Development in Young Children. New York, NY US: Guliford.

Eccleston, C. (1994). Chronic pain and attention: A cognitive approach. British Journal of Clinical Psychology, 33, 535-547.

Eiser, C., \& Havermans, T. (1992). Children's understanding of cancer. Psycho-Oncology, 1, $169-181$. 
Fox, N. (1994). Dynamic cerebral processes underlying emotion regulation. In N.A. Fox (Ed.), The development of emotion regulation: Biological and behavioral considerations (pp. 152-166). Chicago: IL: The University of Chicago Press

Fox, N. \& Calkins, S. (2003). The development of self-control of emotion: Intrinsic and extrinsic influences. Motivation and Emotion, 27(1), 7-26.

Garber, J. \& Dodge, K. (1991). Domains of emotion regulation. In J. Garber \& K. A. Dodge (Eds.), The development of emotion regulation and dysregulation. New York, NY US: Cambridge University Press.

Garner, P. \& Spears, F. (2000). Emotion regulation in low-income preschoolers. Social Development, 9(2), 246-264.

Gray, J. (2004). Integration of emotion and cognitive control. Current Directions in Psychological Science (Wiley-Blackwell), 13(2), 46-48.

Gyurak, A., Goodkind, M., Framer, J., Miller, B., \& Levenson, R. (2012). Executive functions and the down-regulation and up-regulation of emotions. Cognition and Emotion, 26(1), $103-118$.

Jaaniste, T., Hayes, C., \& von Baeyer, C. (2007). Providing children with information about forthcoming medical procedures: A review and synthesis. Clinical Psychology: Science \& Practice, 14(2), 124-143.

Kister \& Patterson, C. (1980). Children's conceptions of the causes of illness: Understanding of contagion and use of immanent justice. Child Development, 51, 839-846.

Koopman, H., Baars, R., Chaplin, J., \& Zwinderman, K. (2004). Illness through the eyes of the child: The development of children's understanding of the causes of illness. Patient Education and Counseling, 55, 363-370. 
Kopp, C. (1989). Regulation of distress and negative emotions: A developmental view. Developmental Psychology, 25(3), 343-354.

Larossa, R. (2005). Grounded theory methods and qualitative family research. Journal of Marriage and Family, 67(4), 837-857.

Lazarus, R. S. (1993). From psychological stress to the emotions: A history of changing outlooks. Annual Review of Psychology, 44(1), 1.

Leerkes, E., Paradise, M., O’Brien, M., \& Calkins, S., (2008). Emotion and cognition processes in preschool children. Merrill-Palmer Quarterly, 54(1), 102-124.

Lewick J. (2013). Psychosocial implications of pediatric surgical hospitalization. Seminars in Pediatric Surgery, 22, 129-133.

Lewis, M. \& Stieben, J. (2004). Emotional regulation in the brain: Conceptual issues and directions for developmental research. Child Development, 75(2), 371-376.

Levetown, M. (2008). Communicating with children and families: From everyday interactions to skills in conveying distressing information. Pediatrics, 121, e1441-e1460.

McIntosh, C., Stephens, C. \& Lyons, A. (2013). "Remember the bubbles hurt you when you cook in the pan": Young children's view of illness causality. Psychology, Health \& Medicine, 18(1), 21-29.

Meyer, S., Raikes, A., Virmani, E., Waters, S., \& Thompson, R. (2014). Parent emotion representation and the socialization of emotion regulation in the family. International Journal of Behavioral Development, 38(2), 164-173.

Morelen, C., Jacob, M., Suveg. C., Jones. A., \& Thomassin, K. (2013). Family emotion expressivity, emotion regulation, and the link to psychopathology: Examination across race. British Journal of Psychology, 104, 149-166. 
Myant, K. \& Williams, J. (2005). Children's concept of health and illness: Understanding of contagious illnesses, non-contagious illnesses and injuries. Journal of Health Psychology, 10(6), 805-819.

Ochner, K. \& Gross, J. (2005). The cognitive control of emotion. Trends in Cognitive Sciences, 9(5), 242-249.

Paterson, J., Moss-Morris, R. \& Butler, S. (1999). The effect of illness experience and demographic factors on children's illness representations. Psychology and Health, 14, $117-129$.

Perrin, E. \& Gerrity, S. (1981). There's a demon in your belly: Children's understanding of illness. Pediatrics, 67(6), 841- 849.

Piaget, J. \& Inhelder, B. (1969). The psychology of the child. New York: NY US: Basic Books, Inc.

Posner, M. \& Rothbart, M. (2000). Developing mechanism of self-regulation. Development and Psychopathology, 12, 427-441.

Redpath, C. \& Rogers, C. (1984). Healthy young children's concepts of hospitals, medical personnel, operations and illness. Journal of Pediatric Psychology, 9(1), 29-40.

Rothbart, M., Scheese, B., Rueda, R., \& Posner, M. (2011). Developing mechanisms for selfregulation in early life. Emotion Review, 3(2), 207-213.

Rokach, A. (2010). The physical, psychosocial, and emotional needs of hospitalized children. Psychology Journal, 7(3), 94-102.

Rushforth, H. (1999). Practitioner review: Communicating with hospitalized children: Review and application of research pertaining to children's understanding of health and illness. Journal of Child Psychology, 40(5), 683-691. 
Shagena, M., Sandler, H., \& Perrin, E. (1988). Concepts of illness and perceptions of control in healthy children and in children with chronic illness. Developmental and Behavioral Pediatrics, 9(5), 252-256.

Schmeichel, B., Volokhov, R., \& Demaree, H. (2008). Working memory capacity and the selfregulation of emotional expression and experience. Journal of Personality and Social Psychology, 95(6), 1526-1540.

Spirito, A., Stark, L., \& Tye, V. (1994). Stressors and coping strategies described during hospitalization by chronically ill children. Journal of Clinical Child Psychology, 23(3), 314-322.

Thompson, R. (1991). Emotional regulation and emotional development. Educational Psychology, 3(4), 269-307.

Vatne, T., Slaughter, L., \& Ruland, C. (2010). How children with cancer communicate and think about symptoms. Journal of Pediatric Oncology Nursing, 27(1), 24-32.

Vohs, K. \& Baumeister, R. (2011). Handbook of self-regulation: Research, theory and application. New York, NY US: Guliford Press.

Walden, T. \& Smith, M. (1997). Emotion regulation. Motivation and Emotion, 21(1), 7-25.

Whaley, B. (2000). Explaining illness to children: Theory, strategies, and future inquiry. In B. B. Whaley (Ed.), Explaining illness: Research, theory, and strategies (pp. 195-207). Mahwah, NJ US: Lawrence Erlbaum Associates Publishers.

Williams, J. \& Binnie, L. (2002). Children's concept of illness: An intervention to improve knowledge. British Journal of Health Psychology, 7, 129-147.

Winner, E. (1986). Where pelicans kiss seals. Psychology Today, 20(8), 24-35. 
Table 1

Descriptive Statistics for Sample

\begin{tabular}{|c|c|c|c|c|c|}
\hline & $n$ & Mean & $S D$ & Minimum & Maximum \\
\hline Participating Child's Age in Years & 26 & 4.75 & .848 & 3.00 & 5.90 \\
\hline Lifetime Frequency of Hospitalization & 26 & 3.62 & 4.06 & 1 & 15 \\
\hline Participating Parent/Guardian's Age in Years & 26 & 34.50 & 8.31 & 25 & 56 \\
\hline
\end{tabular}


Table 2

Descriptive Statistics for General Illness Concept and Behavioral Rating Inventory by Gender

\begin{tabular}{|c|c|c|c|c|c|c|}
\hline \multicolumn{2}{|c|}{ Participating Child's Gender } & \multirow{2}{*}{$\begin{array}{c}n \\
18\end{array}$} & \multirow{2}{*}{$\begin{array}{c}\text { Mean } \\
2.12\end{array}$} & \multirow{2}{*}{$\begin{array}{c}S D \\
.935\end{array}$} & \multirow{2}{*}{$\begin{array}{c}\text { Minimum } \\
.00\end{array}$} & \multirow{2}{*}{$\begin{array}{c}\text { Maximum } \\
3.10\end{array}$} \\
\hline Male & General Illness Concept & & & & & \\
\hline & Emotional Control & 18 & 59.78 & 14.10 & 43.00 & 86.00 \\
\hline & Shift & 18 & $55.00^{*}$ & 13.01 & 40.00 & 83.00 \\
\hline & Inhibit & 18 & $55.61^{*}$ & 12.48 & 38.00 & 83.00 \\
\hline & Flexibility Index & 18 & $58.50^{*}$ & 15.24 & 40.00 & 86.00 \\
\hline & Inhibitory Self Control & 18 & $58.39^{*}$ & 14.13 & 38.00 & 89.00 \\
\hline \multirow[t]{6}{*}{ Female } & General Illness Concept & 8 & 2.94 & .318 & 2.42 & 3.43 \\
\hline & Emotional Control & 8 & 51.50 & 7.87 & 38.00 & 63.00 \\
\hline & Shift & 8 & $49.75^{*}$ & 6.18 & 43.00 & 62.00 \\
\hline & Inhibit & 8 & $55.63^{*}$ & 7.13 & 43.00 & 66.00 \\
\hline & Flexibility Index & 8 & $50.88^{*}$ & 8.03 & 39.00 & 65.00 \\
\hline & Inhibitory Self Control & 8 & $54.25^{*}$ & 6.39 & 45.00 & 64.00 \\
\hline
\end{tabular}

Note. ${ }^{*} p<.05, * * p<.01$. Behavioral Rating Inventory in T-values 
Table 3

Descriptive Statistics for Behavioral Rating Inventory T-values

\begin{tabular}{lccccc}
\hline & $n$ & Mean & $S D$ & Minimum & Maximum \\
\hline Emotional Control & & & & \\
Shift & 26 & 57.23 & 12.95 & 38.00 & 86.00 \\
Inhibit & 26 & 53.38 & 11.49 & 40.00 & 83.00 \\
Flexibility & 26 & 55.62 & 10.96 & 38.00 & 83.00 \\
Inhibitory Self Control & 26 & 56.15 & 13.74 & 39.00 & 86.00 \\
& 26 & 57.12 & 12.28 & 38.00 & 89.00 \\
\hline
\end{tabular}


Table 4

Definitions and Frequencies of Verbal Descriptions of Germs Classifications

\begin{tabular}{|c|c|c|c|c|c|}
\hline Level & $n$ & $\%$ & $\begin{array}{l}\text { Assigned } \\
\text { Rule }\end{array}$ & Definition & Prototype Responses \\
\hline 1 & 4 & 15.40 & No response & Inability to respond & "I don't know" \\
\hline 2 & 8 & 30.80 & Bad & $\begin{array}{l}\text { Explanations referring to the } \\
\text { term bad }\end{array}$ & "They are bad and mean" \\
\hline 3 & 2 & 7.70 & Dirty & Features describing dirt or dirty & $\begin{array}{l}\text { "They are outside in the mud and the dirt"; "Germs are } \\
\text { yucky stuff in your body"; "Green stuff on the floor"; "You } \\
\text { got to stick them in a garbage truck" }\end{array}$ \\
\hline 4 & 6 & 23.10 & Disease & $\begin{array}{l}\text { Refers to something that makes } \\
\text { someone ill }\end{array}$ & $\begin{array}{l}\text { "I don't give germs to anyone. I wash my hands"; "They bite } \\
\text { your belly and make you hurt"; "They make your body } \\
\text { really sick" }\end{array}$ \\
\hline 5 & 6 & 23.10 & Other & $\begin{array}{l}\text { Describes a concept that does } \\
\text { not fall within the specified } \\
\text { categories }\end{array}$ & $\begin{array}{l}\text { "They will eat you"; I think the doctor will protect you"; "It } \\
\text { looks like my mouth"; "They give allergies"; "You can't see } \\
\text { it but they make food taste gross"; "People have them } \\
\text { inside" }\end{array}$ \\
\hline
\end{tabular}


Table 5

Pearson Correlation Coefficients for General Illness Concept and Behavioral Rating Inventory

\begin{tabular}{|c|c|c|c|c|c|c|}
\hline & GICI & $\mathrm{EC}$ & $\mathrm{S}$ & $\mathrm{I}$ & $\mathrm{F}$ & ISC \\
\hline General Illness Concept Interview & - & -.32 & $-.35 *$ & $-.42 *$ & $-.37 *$ & $-.45^{*}$ \\
\hline Emotional Control & & - & $.81 * *$ & $.54 * *$ & $.90 * *$ & $.79 * *$ \\
\hline Shift & & & - & $.61 * *$ & $.97 * *$ & $.79 *$ \\
\hline Inhibit & & & & - & $.63 * *$ & $.93 * *$ \\
\hline Flexibility & & & & & - & $.85^{* *}$ \\
\hline Inhibitory Self Control & & & & & & - \\
\hline
\end{tabular}

Note. ${ }^{*} p<.05, * * p<.01$. GICI = General Illness Concept Interview; EC = Emotional Control; S = Shift; I = Inhibit; F = Flexibility; ISC $=$ Inhibitory Self-Control. 
Table 6

Independent Sample t-Test for Initial and Extensive Exposure to Hospitalization Category for Variables of Interest

\begin{tabular}{|c|c|c|c|c|c|c|c|c|}
\hline & \multicolumn{2}{|c|}{ Initial Exposure } & \multicolumn{2}{|c|}{ Extensive Exposure } & \multirow{3}{*}{$t$} & \multirow{3}{*}{$d f$} & \multirow{3}{*}{$\begin{array}{c}\text { Mean } \\
\text { Difference }\end{array}$} & \multirow{3}{*}{$\begin{array}{l}\text { Std. Error } \\
\text { Difference }\end{array}$} \\
\hline & $M$ & $S D$ & $M$ & $S D$ & & & & \\
\hline & & & & & & & & \\
\hline General Illness Interview & 2.68 & .561 & 2.02 & 1.06 & 2.04 & 24 & .664 & .326 \\
\hline Emotional Control & 52.00 & 10.32 & 63.33 & 13.41 & $-2.43 * *$ & 24 & -11.33 & 4.66 \\
\hline Shift & 47.93 & 5.06 & 59.75 & 13.69 & $-3.01 * *$ & 24 & -11.82 & 3.93 \\
\hline Inhibit & 53.75 & 9.53 & 58.00 & 12.42 & -1.03 & 24 & -4.43 & 4.31 \\
\hline Flexibility & 49.64 & 8.12 & 63.75 & 15.30 & $-2.99 * *$ & 24 & -14.11 & 4.71 \\
\hline Inhibitory Self Control & 53.14 & 9.84 & 61.75 & 13.60 & -1.87 & 24 & -8.61 & 4.61 \\
\hline
\end{tabular}

Note. ${ }^{*} p<.05, * * p<.01$ 
Table 7

Definitions and Frequencies of General Illness Concept Interview Classifications

\begin{tabular}{|c|c|c|c|c|c|}
\hline Level & $n$ & $\%$ & Assigned Rule & Definition & Prototype Responses \\
\hline 0 & 19 & 9.90 & $\begin{array}{l}\text { No response or off-task } \\
\text { inappropriate response }\end{array}$ & Refusal to respond & $\begin{array}{l}\text { "I like SpongeBob"; "Wheels are } \\
\text { shinny"; "Like a piggy" }\end{array}$ \\
\hline 1 & 26 & 13.50 & Inability to respond & Do not know & "I don't know" \\
\hline 2 & 54 & 28.10 & Global response & A phenomenon & $\begin{array}{l}\text { "You're sad when you're sick"; } \\
\text { "Throwing rocks"; "Coughing and } \\
\text { sneezing"; "Rug burn" }\end{array}$ \\
\hline 3 & 48 & 25.00 & Concrete rules & Concrete external causes & $\begin{array}{l}\text { "Eating too much"; "You take } \\
\text { medicine"; "You get goose bumps" }\end{array}$ \\
\hline 4 & 40 & 20.80 & Internalization & Internalization of agents & $\begin{array}{l}\text { "Staying healthy"; "Washing your } \\
\text { hands"; "When it is raining out and } \\
\text { you don't have a hat"; "Eating } \\
\text { good food" }\end{array}$ \\
\hline 5 & 5 & 2.60 & Interaction & $\begin{array}{l}\text { Internalization of agent and } \\
\text { understands bodily response }\end{array}$ & $\begin{array}{l}\text { "Helps the doctor make your body } \\
\text { healthy"; "Medicine kills germs"; } \\
\text { "It heals your body" }\end{array}$ \\
\hline 6 & 0 & 0.00 & Generalized Principles & $\begin{array}{l}\text { Mechanisms of the processes } \\
\text { of illness causation, prevention } \\
\text { and treatment }\end{array}$ & $\begin{array}{l}\text { "Germs take away food from the } \\
\text { body and then the body has nothing } \\
\text { to use for power"; "You breathe in } \\
\text { something bad from another person } \\
\text { and it breaks down your body" }\end{array}$ \\
\hline
\end{tabular}




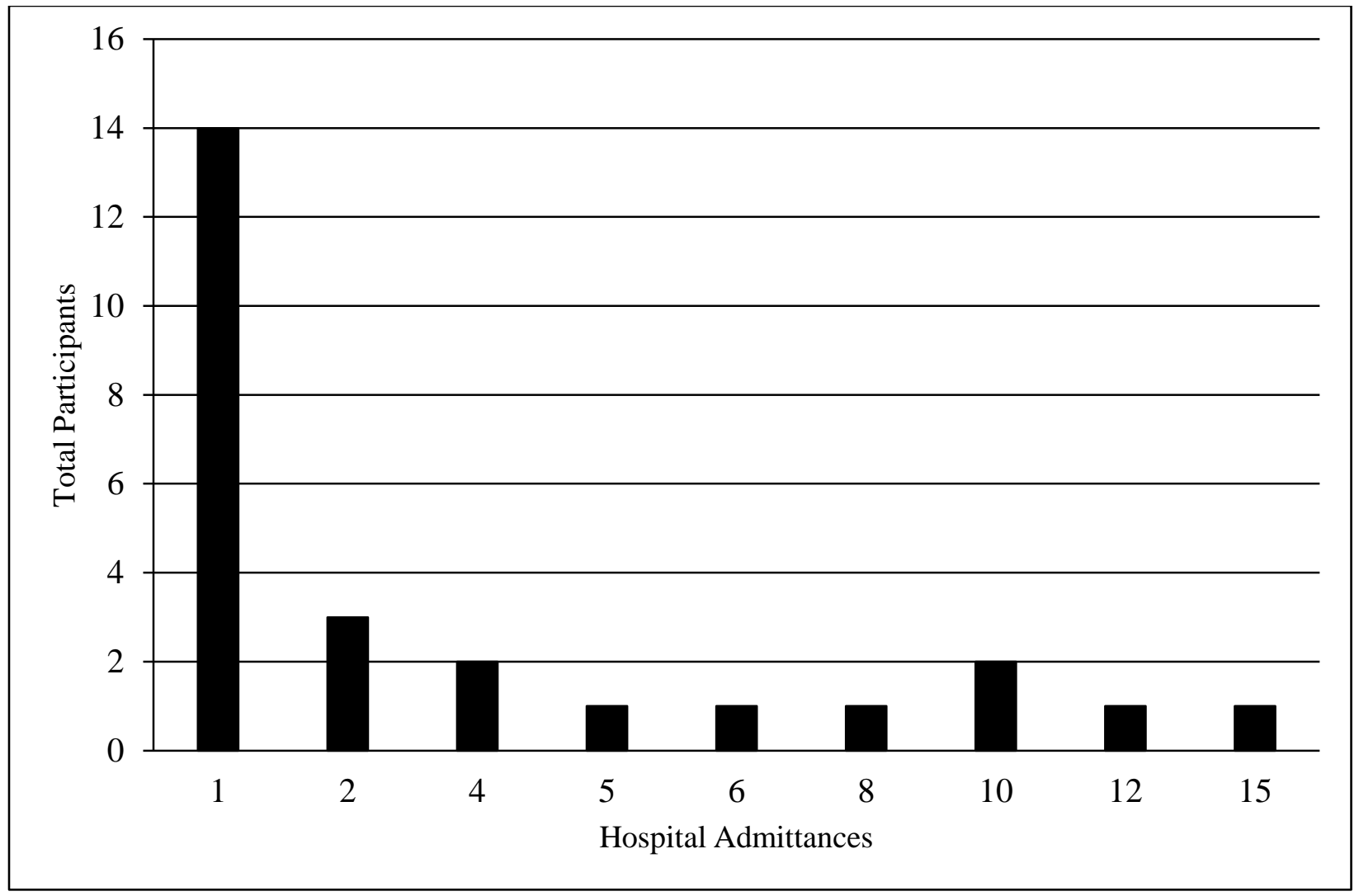

Figure 1

Total Hospital Admittances of Participating Pediatric Patients 


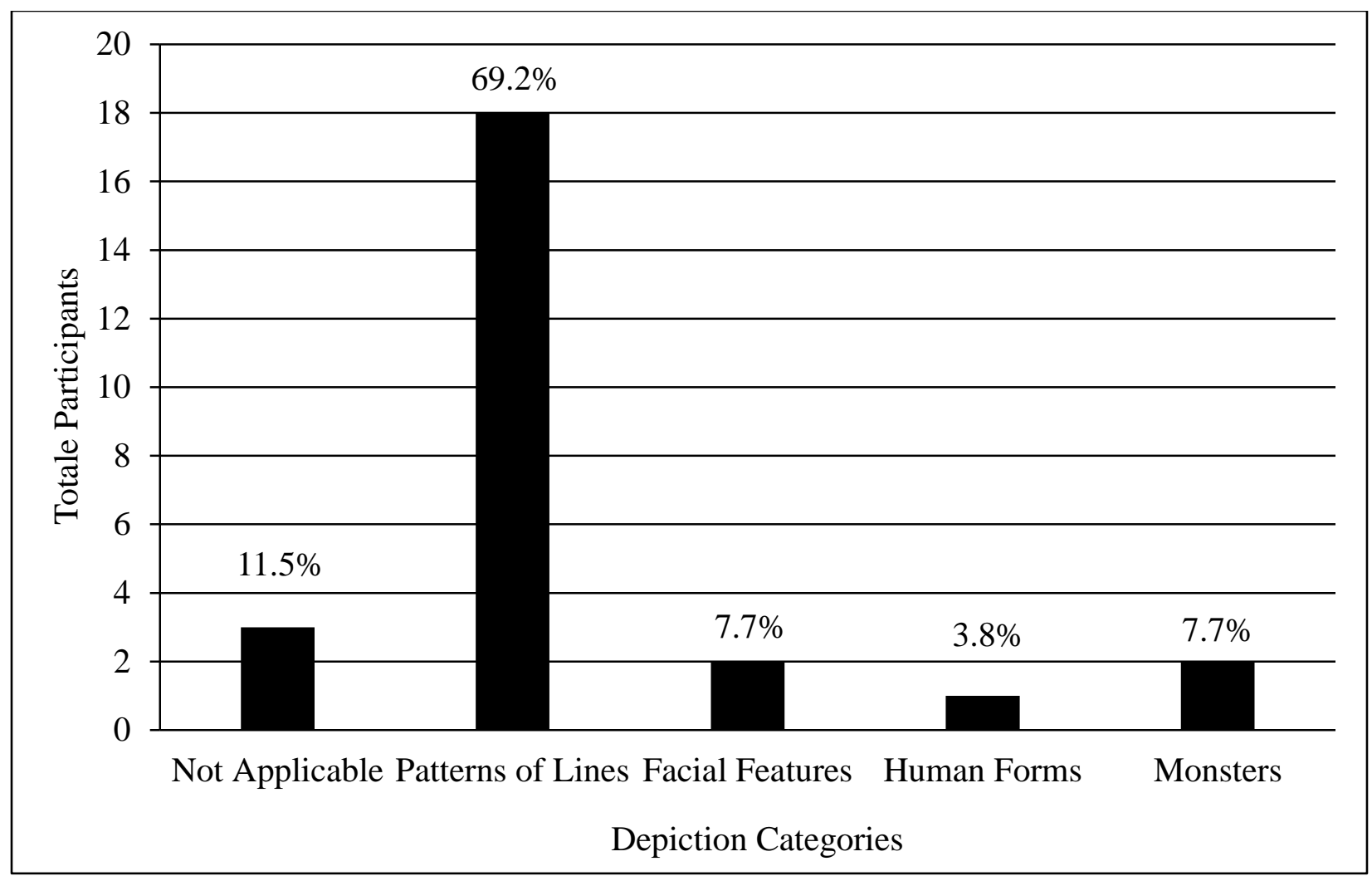

Figure 2

Frequencies of Depiction of Germ Classifications 
Figure 3

Sample Depictions of a Germ

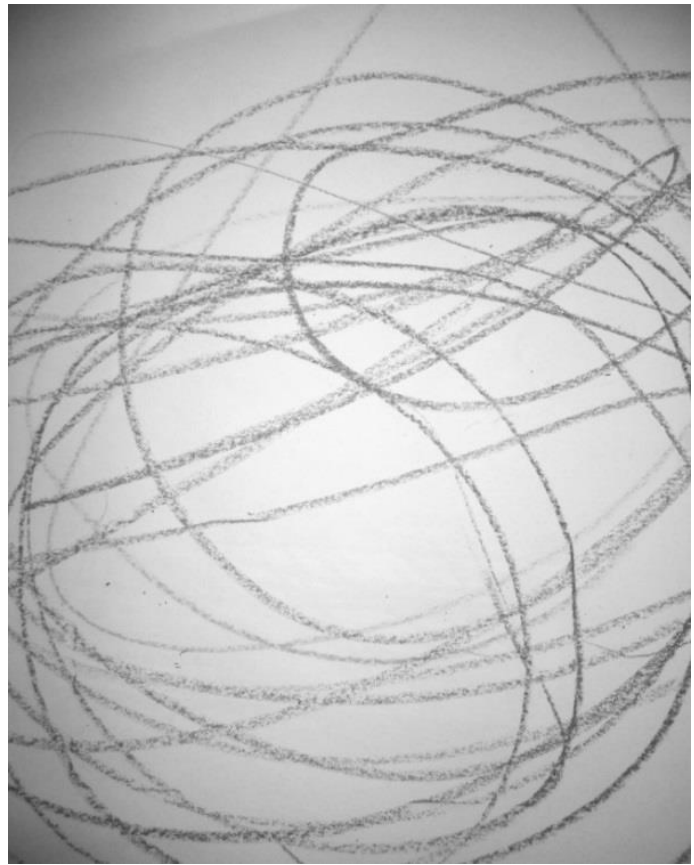

Patterns of Lines

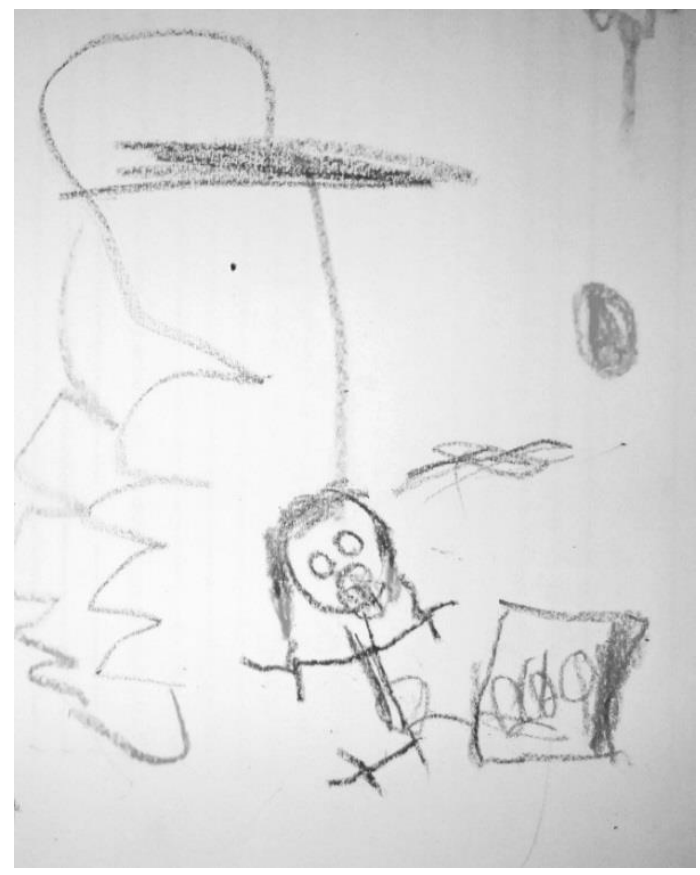

Human Figures

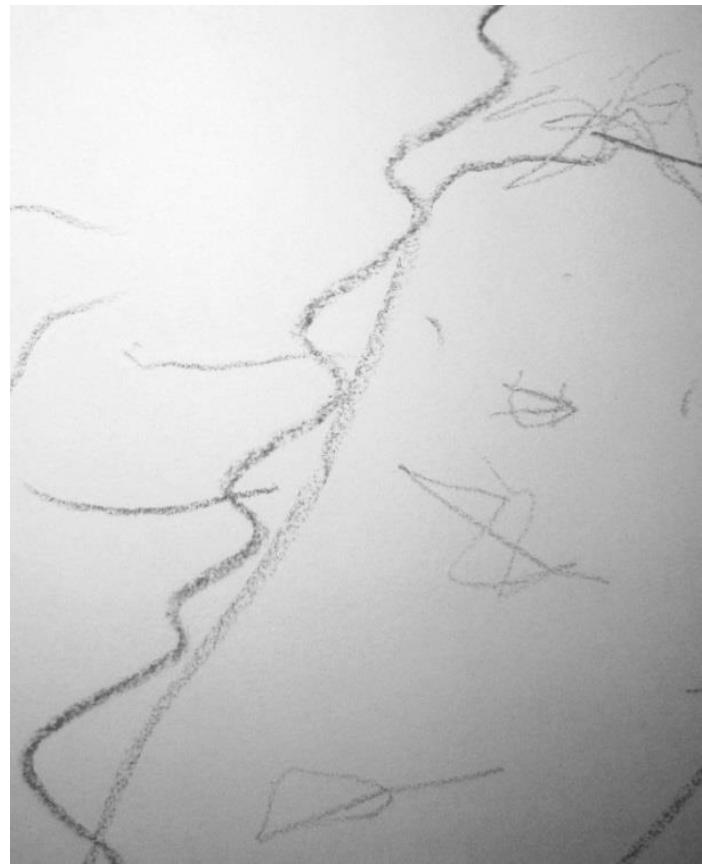

Facial Features

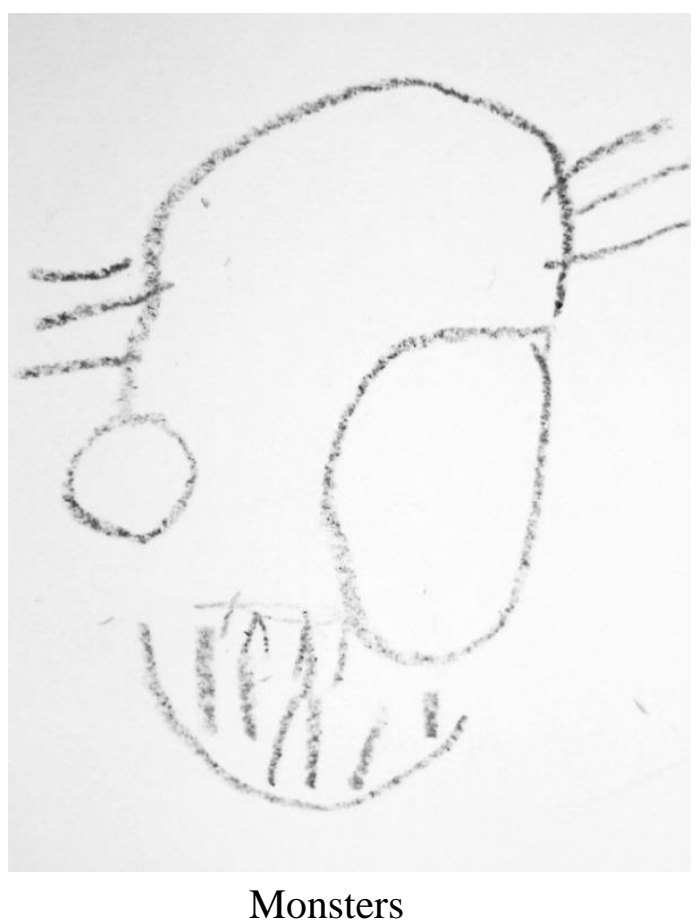


Figure 4

\section{Supplemental Sample Depiction of a Germ}

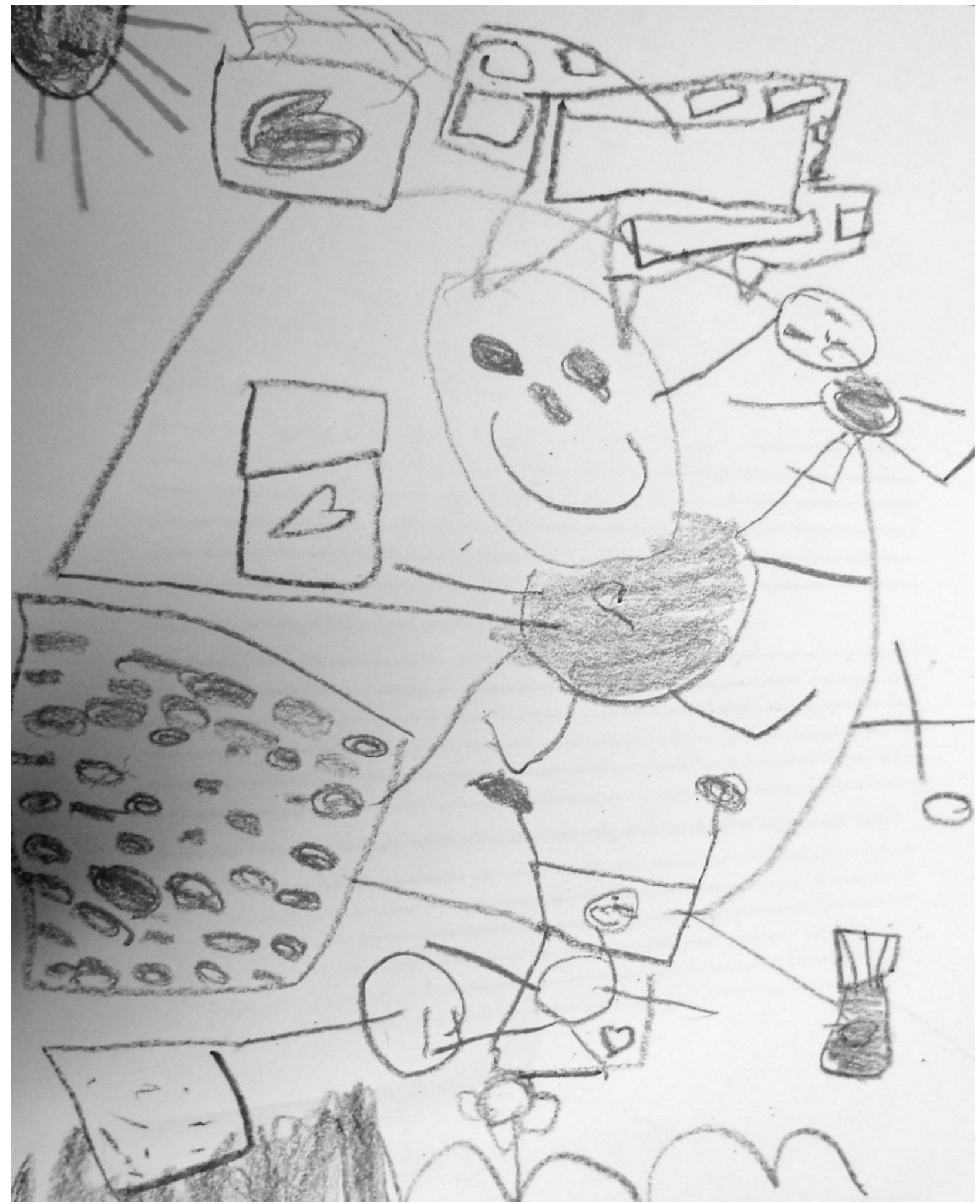




\section{Appendix A \\ General Demographic Questionnaire}

Please disclose the following information regarding your child with a check mark:

Child's Date of Birth: (MM/DD/YYYY)

\section{Gender:}
[] Male
[ ] Female
[ ] Unidentified

Other than the birth of your child, how many times in his or her life has your child been hospitalized overnight?

What is your relationship to the child?

[ ] Mother [ ] Father [ ] Grandparent [ ] Legal Guardian

[ ] Other, please specify

Your Age: 


\section{Appendix B}

\section{The Behavioral Rating Inventory of Executive Function-Preschool}

Child's Name

Relationship to Child: $\square$ Mother $\quad \square$ Father $\quad \square$ Teacher $\quad \square$ Other

During the past 6 months, how often has each of the following behaviors been a problem?

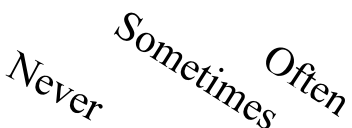

Overreacts to small problems

$\mathrm{N} \quad \mathrm{S} \quad \mathrm{O}$

Is unaware of how his/her behavior affects or bothers others

Becomes upset with new situations

$\mathrm{N} \quad \mathrm{S} \quad \mathrm{O}$

Has explosive, angry outbursts

$\mathrm{N} \quad \mathrm{S} \quad \mathrm{O}$

Does not stop laughing at funny things or events when others stop

$\mathrm{N} \quad \mathrm{S} \quad \mathrm{O}$

Has trouble adjusting to new people (such as babysitter, teacher, friend, or day $\quad \mathrm{N} \quad \mathrm{S} \quad \mathrm{O}$ care worker)

Becomes upset too easily

$\begin{array}{lll}\mathrm{N} & \mathrm{S} & \mathrm{O} \\ \mathrm{N} & \mathrm{S} & \mathrm{O} \\ \mathrm{N} & \mathrm{S} & \mathrm{O}\end{array}$

Is upset by a change in plans or routine (for example, order of daily activities, adding last minute errands to schedule, change in driving route to store)

$\begin{array}{llll}\text { Has outbursts for little reason } & \mathrm{N} & \mathrm{S} & \mathrm{O} \\ \text { Acts wilder or sillier than others in groups (such as birthday parties, play } & \mathrm{N} & \mathrm{S} & \mathrm{O}\end{array}$
group)

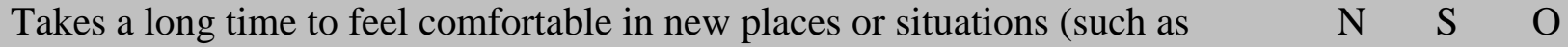
visiting distant relatives or new friends)

Mood changes frequently $\mathrm{N} \quad \mathrm{S} \quad \mathrm{O}$

Is fidgety, restless, or squirmy

$\mathrm{N} \quad \mathrm{S} \quad \mathrm{O}$

$\begin{array}{llll}\text { Is bothered by loud noises, bright lights, or certain smells } & \mathrm{N} & \mathrm{S} & \mathrm{O}\end{array}$

Small events trigger big reactions $\quad N \quad S \quad O$

Is impulsive

Is disturbed by changes in the environment (such as new furniture, things in $\quad \mathrm{N} \quad \mathrm{S} \quad \mathrm{O}$ room moved around, or new clothes)

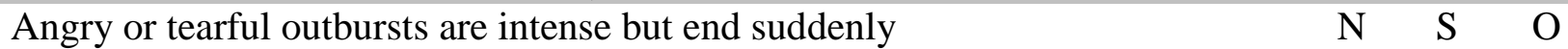

$\begin{array}{llll}\text { Does not notice when his/her behavior causes negative reactions } & \mathrm{N} & \mathrm{S} & \mathrm{O}\end{array}$

Has trouble changing activities

Reacts more strongly to situations than other children

$\mathrm{N} \quad \mathrm{S} \quad \mathrm{O}$

Does not realize that certain actions bother others

$\mathrm{N} \quad \mathrm{S} \quad \mathrm{O}$

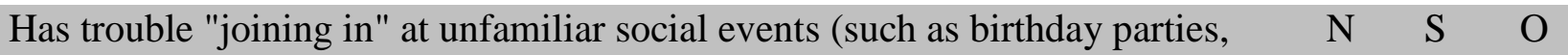
picnics, holiday gatherings)

Is easily overwhelmed or overstimulated by typical daily activities

Gets out of control more than playmates

\begin{tabular}{lll}
$\mathrm{N}$ & $\mathrm{S}$ & $\mathrm{O}$ \\
$\mathrm{N}$ & $\mathrm{S}$ & $\mathrm{O}$ \\
$\mathrm{N}$ & $\mathrm{S}$ & $\mathrm{O}$ \\
$\mathrm{N}$ & $\mathrm{S}$ & $\mathrm{O}$ \\
$\mathrm{N}$ & $\mathrm{S}$ & $\mathrm{O}$ \\
$\mathrm{N}$ & $\mathrm{S}$ & $\mathrm{O}$ \\
\hline
\end{tabular}

Resists change of routine, foods, places, etc.

After having a problem, will stay disappointed for a long time

Talks or plays too loudly

Acts overwhelmed or overstimulated in crowded, busy situations (such as lots 
of noise, activity, or people)

Acts too wild or out of control

$\begin{array}{lll}\mathrm{N} & \mathrm{S} & \mathrm{O}\end{array}$

Has trouble putting the brakes on his/her actions even after being asked

$\mathrm{N} \quad \mathrm{S} \quad \mathrm{O}$

Completes tasks or activities too quickly

$\mathrm{N} \quad \mathrm{S} \quad \mathrm{O}$

Gets easily sidetracked during activities

$\mathrm{N} \quad \mathrm{S} \quad \mathrm{O}$

Becomes too silly

$\mathrm{N} \quad \mathrm{S} \quad \mathrm{O}$

Plays carelessly or recklessly in situations where he/she could be hurt (such as

$\mathrm{N} \quad \mathrm{S} \quad \mathrm{O}$ playground, swimming pool) 
Appendix C

The General Illness Concept Interview

How do children know when they are sick?

How do children get sick?

How can children keep from getting sick?

Some children get stomach aches when they are sick. How do children get stomach aches? 
Sometimes when children get sick they get little bumps or spots on their skin. How do kids get those bumps or spots?

When children are sick, how can they get better?

Sometimes when children get sick they have to take medicine. How does medicine work? 


\section{Appendix D}

Subset of Banks Questionnaire

What is a germ?

Can you draw a picture of a germ? 